\title{
HOLOMORPHIC ONE-FORMS WITHOUT ZEROS ON THREEFOLDS
}

\author{
FENG HAO AND STEFAN SCHREIEDER
}

\begin{abstract}
We show that a smooth complex projective threefold admits a holomorphic one-form without zeros if and only if the underlying real 6 -manifold is a $C^{\infty}$-fibre bundle over the circle, and we give a complete classification of all threefolds with that property. Our results prove a conjecture of Kotschick in dimension three.
\end{abstract}

\section{INTRODUCTION}

1.1. Holomorphic one-forms and fibre bundles over the circle. For a smooth complex projective variety $X$ we may consider the following conditions:

(A) $X$ admits a holomorphic one-form without zeros;

(B) $X$ admits a real closed 1-form without zeros; or, by Tischler's theorem [Ti70] equivalently, the underlying differentiable manifold is a $C^{\infty}$-fibre bundle over the circle.

Note that while $(\mathrm{A})$ is an algebraic condition on $X$, condition (B) is a purely topological one which by [Ti70] characterizes the smooth manifold which underlies $X$ as $[0,1] \times M / \sim$, where $M$ is a closed manifold of real dimension $2 \operatorname{dim}(X)-1$ and where $0 \times M$ is identified with $1 \times M$ via some diffeomorphism of $M$.

While $(\mathrm{A}) \Rightarrow(\mathrm{B})$ is clear, Kotschick conjectured [Kot13] that both condition might be equivalent to each other. In [Sch19], the second author developed an approach to this conjecture, showing that (B) implies

(C) there is a holomorphic one-form $\omega \in H^{0}\left(X, \Omega_{X}^{1}\right)$ such that for any finite étale cover $\tau: X^{\prime} \rightarrow X$, the sequence

$$
H^{i-1}\left(X^{\prime}, \mathbb{C}\right) \stackrel{\wedge \omega^{\prime}}{\longrightarrow} H^{i}\left(X^{\prime}, \mathbb{C}\right) \stackrel{\wedge \omega^{\prime}}{\longrightarrow} H^{i+1}\left(X^{\prime}, \mathbb{C}\right)
$$

given by cup product with $\omega^{\prime}:=\tau^{*} \omega$ is exact for all $i$.

Moreover, all three conditions above coincide in dimension two [Sch19, Theorem 1.3]. In this paper, we address the much more difficult case of threefolds.

Theorem 1.1. Let $X$ be a smooth complex projective threefold. Then all three conditions above are equivalent to each other: $(A) \Leftrightarrow(B) \Leftrightarrow(C)$.

Date: June 18th, 2019.

2010 Mathematics Subject Classification. primary 14F45, 14J30, 32Q55; secondary 32Q57.

Key words and phrases. Topology of algebraic varieties, one-forms, minimal model program, classification, generic vanishing, threefolds. 


\subsection{Classifying threefolds whose underlying 6-manifolds fibre over the circle.}

Theorem 1.1 follows from the following strong classification result.

Theorem 1.2. If $X$ is a smooth complex projective threefold, any of the conditions (A), $(B)$ and $(C)$ is equivalent to the following:

(D) the minimal model program for $X$ yields a birational morphism $\sigma: X \rightarrow X^{\mathrm{min}}$ to a smooth projective threefold $X^{\mathrm{min}}$, such that:

(1) $\sigma: X \rightarrow X^{\mathrm{min}}$ is a sequence of blow-ups along smooth elliptic curves that are not contracted via the natural map to the Albanese variety $\operatorname{Alb}\left(X^{\mathrm{min}}\right)$.

(2) There is a smooth morphism $\pi: X^{\mathrm{min}} \rightarrow A$ to a positive-dimensional abelian variety $A$.

(3) If $\kappa(X) \geq 0$, then $X^{\mathrm{min}}$ is the quotient of a product $S^{\prime} \times A^{\prime}$ by a free and diagonal action of a finite group $G$, where $S^{\prime}$ is smooth projective, $A^{\prime}$ is an abelian variety that is étale over $A \simeq A^{\prime} / G$ and $\pi$ is induced by the projection $S^{\prime} \times A^{\prime} \rightarrow A^{\prime}$.

(4) If $\kappa(X)=-\infty$, then one of the following holds:

(i) $X^{\text {min }}$ admits a smooth del Pezzo fibration over an elliptic curve;

(ii) $X^{\text {min }}$ has the structure of a conic bundle $f: X^{\text {min }} \rightarrow S$ over a smooth projective surface $S$ which satisfies $(A) \Leftrightarrow(B) \Leftrightarrow(C)$. Moreover, $f$ is either smooth, or $A$ is an elliptic curve and the degeneration locus of $f$ is a disjoint union of smooth elliptic curves on $S$ which are étale over $A$ (via the map $S \rightarrow A$ induced by $\pi$ ).

Note that $(\mathrm{D}) \Rightarrow(\mathrm{A})$, because a general one-form on $X$ has no zeros by (D1) and (D2). Since $(A) \Rightarrow(B)$ is clear and $(B) \Rightarrow(C)$ is known by [Sch19], in order to prove Theorems 1.1 and 1.2, it thus suffices to show $(\mathrm{C}) \Rightarrow(\mathrm{D})$. In the course of our proof, we will obtain the following refined version of $(\mathrm{A}) \Leftrightarrow(\mathrm{C})$.

Theorem 1.3. Let $X$ be a smooth complex projective threefold and let $\omega \in H^{0}\left(X, \Omega_{X}^{1}\right)$ be a holomorphic one-form on $X$. Then the following are equivalent:

(1) $\omega$ has no zero on $X$;

(2) for any étale cover $\tau: X^{\prime} \rightarrow X$, the sequence given by cup product with $\omega^{\prime}:=\tau^{*} \omega$

$$
H^{i-1}\left(X^{\prime}, \mathbb{C}\right) \stackrel{\wedge \omega^{\prime}}{\longrightarrow} H^{i}\left(X^{\prime}, \mathbb{C}\right) \stackrel{\wedge \omega^{\prime}}{\longrightarrow} H^{i+1}\left(X^{\prime}, \mathbb{C}\right)
$$

is exact for all $i$.

The implication $(1) \Rightarrow(2)$ is a result of Green and Lazarsfeld which holds in arbitrary dimensions, see [GL87, Proposition 3.4]. The converse implication (2) $\Rightarrow(1)$ has previously been proven in dimension two by the second author in [Sch19, Theorem 1.3].

Theorem 1.3 has the following interesting consequence. 
Corollary 1.4. For a holomorphic one-form $\omega \in H^{0}\left(X, \Omega_{X}^{1}\right)$ on a smooth complex projective threefold $X$, the condition that $\omega$ has no zeros on $X$ is a topological one which depends only on the cohomology class $[\omega] \in H^{*}(X, \mathbb{C})$ of $\omega$ and the homotopy type of $X$.

By the above corollary, if $\omega$ is a holomorphic one-form without zeros on a smooth projective threefold $X$, then for any smooth projective threefold $X^{\prime}$ which is deformation equivalent to $X$, and for any one-form $\omega^{\prime} \in H^{0}\left(X^{\prime}, \Omega_{X^{\prime}}^{1}\right)$ that is obtained via parallel transport of $\omega$ with respect to some path, $\omega^{\prime}$ has no zeros on $X^{\prime}$. This is interesting already in the case where $X=X^{\prime}$.

1.3. Around a theorem of Popa and Schnell. Recall that items (D1) and (D2) imply condition (A). Hence, Theorem 1.2 shows in particular that (D1) and (D2) imply conditions (D3) and (D4). For this reason, Theorem 1.2 has the following consequence; we give the details of the argument in Section 9 below.

Corollary 1.5. Let $f: X \rightarrow A$ be a smooth morphism from a smooth projective threefold $X$ to an abelian variety $A$. If $\kappa(X) \geq 0$, then there is a smooth projective threefold $X^{\prime}$ with the structure of an analytic fibre bundle $f^{\prime}: X^{\prime} \rightarrow A$ over $A$, such that $X$ and $X^{\prime}$ are birational over $A$.

Passage to a birational model of $X$ is necessary; the example of suitable minimal del Pezzo fibrations over elliptic curves shows that the assumption $\kappa(X) \geq 0$ is necessary as well.

By [Ue75, Theorem 15.1], the Kodaira dimension is additive for analytic fibre bundles and so, in the situation of Corollary 1.5, we have $\kappa(X)=\kappa(F)$, where $F$ denotes a fibre of $f$. Hence, $\kappa(X) \leq \operatorname{dim}(F)$, which is a special case of a celebrated result of Popa and Schnell [PS14] (with earlier results in [Za97, LZ05, HK05]), who showed that a smooth projective variety $X$ has Kodaira dimension $\kappa(X) \leq \operatorname{dim}(X)-d$, if it admits a $d$-dimensional linear subspace $V \subset H^{0}\left(X, \Omega_{X}^{1}\right)$ of holomorphic one-forms such that any nonzero $\omega \in V$ has no zeros on $X$.

The results in this paper lead us to the following two conjectures, which by the aforementioned additivity of Kodaira dimensions in analytic fibre bundles [Ue75, Theorem 15.1] would generalize Popa-Schnell's result.

Conjecture 1.6. Let $X$ be a smooth projective variety which admits a holomorphic oneform without zeros. Then $X$ is birational to a smooth projective variety $X^{\prime}$ which admits a smooth morphism $X^{\prime} \rightarrow A$ to a positive-dimensional abelian variety $A$.

Conjecture 1.7. Let $f: X \rightarrow A$ be a smooth morphism from a smooth projective variety $X$ to an abelian variety $A$. If $\kappa(X) \geq 0$, then, up to birational equivalence, $f$ is an analytic fibre bundle. 
Conjectures 1.6 and 1.7 hold for surfaces by [Sch19, Corollary 3.2] and for threefolds by item (D2) in Theorem 1.2 and Corollary 1.5. If the fibres of $f$ are of general type, a weak form of Conjecture 1.7 had been proven by Popa-Schnell in arbitrary dimensions, see [PS14, Corollary 3.2]. Moreover, [PS14, Corollary 3.2] easily implies Conjecture 1.7 if the fibres of $f$ are curves.

1.4. Why one-forms? Theorem 1.2 yields a complete classification of all smooth complex projective threefolds with a one-form without zeros, and Theorem 1.1 shows that this is in fact a topological property. It is natural to wonder if such a classification is possible also for forms of higher degree. For top differential forms, this essentially amounts to classifying Calabi-Yau threefolds, and it is a famous open problem to show that such varieties come in finitely many topological types. The remaining case is that of two-forms without zeros. Two-forms on threefolds have previously been studied by Campana and Peternell [CaPe00] who found infinitely many examples of smooth projective threefolds of general type (see e.g. [CaPe00, Example 1.3.3]) which carry two-forms without zeros. This suggests that a classification is probably impossible in this case.

1.5. A remark on the Kähler case. It is conceivable that the methods of this paper allow to prove analogues of Theorems 1.1, 1.2 and 1.3 also in the case of Kähler threefolds. The main technical difficulty that one has to overcome is the fact that short exact sequences of abelian varieties always split after étale cover, while this is in general not true for short exact sequences of arbitrary complex tori. As a consequence, item (D3) in Theorem 1.2 does not remain true in the Kähler setting, but we expect that under the Kähler assumption, one can still prove that in item (D3) there is a smooth morphism as in item (D2) which is in fact an analytic fibre bundle.

Conventions and notation. We work over the field of complex numbers. A variety is an integral separated scheme of finite type over $\mathbb{C}$. A minimal model is a projective variety $X$ with terminal $\mathbb{Q}$-factorial singularities such that $K_{X}$ is nef.

\section{Preliminaries}

2.1. Analytic fibre bundles. A proper morphism $f: X \rightarrow S$ of complex manifolds (or smooth complex projective varieties), is an analytic fibre bundle, if it is locally isomorphic to a product of the base with a typical fibre $F$. The isomorphism type of $f$ is determined by a cocycle in $H^{1}(S, \operatorname{Aut}(F))$. Moreover, by a well-known result of Fischer and Grauert [FG65], a proper morphism $f: X \rightarrow S$ of complex manifolds is an analytic fibre bundle if and only if it is isotrivial, i.e. all fibres are isomorphic to each other. 
2.2. Galois covers and quasi-étale quotients. A finite morphism $f: X^{\prime} \rightarrow X$ between normal varieties is quasi-étale if it is étale in codimension one, see e.g. [GKP16]; if $X$ is smooth, then this implies that $f$ is étale. In particular, $f$ is ramified at most at the singular points of $X$.

A variety $X$ is a finite (quasi-)étale quotient of a variety $X^{\prime}$, if there is a finite subgroup $G \subset \operatorname{Aut}\left(X^{\prime}\right)$ with $X=X^{\prime} / G$ and such that the quotient map $X^{\prime} \rightarrow X$ is (quasi-)étale. We call a finite (quasi-)étale morphism $\tau: X^{\prime} \rightarrow X$ Galois, if it is isomorphic to a finite (quasi-)étale quotient as above. It is a well-known fact that any finite (quasi-)étale cover can be dominated by one that is Galois, see e.g. [GKP16, Theorem 3.7]:

Theorem 2.1. Any finite quasi-étale morphism $f: X \rightarrow Y$ of normal quasi-projective varieties can be dominated by a finite quasi-étale morphism $g: X^{\prime} \rightarrow X$, such that $f \circ g: X^{\prime} \rightarrow Y$ is Galois.

2.3. Basic properties of condition (C). In [Sch19, Theorems 1.2 and 1.5], the second author proved the following two theorems, which are the starting point of our investigation.

Theorem 2.2 ([Sch19]). For any compact Kähler manifold $X$, we have $(B) \Rightarrow(C)$.

Theorem 2.3 ([Sch19]). Let $X$ be a compact Kähler manifold with a holomorphic oneform $\omega$ such that the complex $\left(H^{*}(X, \mathbb{C}), \wedge \omega\right)$ given by cup product with $\omega$ is exact. Then the analytic space $Z(\omega)$ given by the zeros of $\omega \in H^{0}\left(X, \Omega_{X}^{1}\right)$ has the following properties.

(1) For any connected component $Z \subset Z(\omega)$ with $d=\operatorname{dim} Z$,

$$
H^{d}\left(Z,\left.\omega_{X}\right|_{Z}\right)=0
$$

In particular, $\omega$ does not have any isolated zero.

(2) If $f: X \rightarrow A$ is a holomorphic map to a complex torus $A$ such that $\omega \in f^{*} H^{0}\left(A, \Omega_{A}^{1}\right)$, then $f(X) \subset A$ is fibred by tori.

We will also use the following observations.

Lemma 2.4. Let $X$ be a compact Kähler manifold and let $f: X \rightarrow A$ be a morphism to a complex torus $A$ which is generated by the image $f(X)$. Assume that there is a oneform $\alpha \in H^{0}\left(A, \Omega_{A}^{1}\right)$, such that $\left(H^{*}(X, \mathbb{C}), \wedge f^{*} \alpha\right)$ is exact. If there is a prime divisor $D \subset X$ with $\operatorname{dim}(f(D))=0$, then $A$ is an elliptic curve and $D$ is numerically equivalent to some rational multiple of a general fibre of $f$.

Proof. Since $\operatorname{dim}(f(D))=0,[D] \wedge f^{*} \alpha=0$ and so by exactness of $\wedge f^{*} \alpha$ we get

$$
[D]=\bar{\beta} \wedge f^{*} \alpha
$$


for some $\beta \in H^{0}\left(X, \Omega_{X}^{1}\right)$. Since $[D]$ is integral, $\beta$ must be in the image of $f^{*}$. Hence,

$$
[D] \in f^{*} H^{2}(A, \mathbb{Q}) .
$$

Since $f(D)$ is a point, $f(X)$ must be a curve and $[D]$ must be a rational multiple of the class of a fibre of $X \rightarrow f(X)$. Since $f(X)$ is a curve, its normalization is an elliptic curve by exactness of $\left(H^{*}(X, \mathbb{C}), \wedge f^{*} \alpha\right)$. Since $f(X) \subset A$, it must be a smooth elliptic curve and since it generates $A$, the latter is an elliptic curve as well. This concludes the lemma.

Corollary 2.5. In the situation of Lemma 2.4, the Stein factorization of $f$ yields a morphism $g: X \rightarrow E$ to an elliptic curve $E$ with irreducible fibres.

Proof. Since $A$ is an elliptic curve, the Stein factorization of $f$ yields a morphism $g: X \rightarrow$ $C$ to a smooth projective curve $C$. By assumptions, there is a one-form $\beta \in H^{0}\left(C, \Omega_{C}^{1}\right)$ such that $\left(H^{*}(X, \mathbb{C}), \wedge g^{*} \beta\right)$ is exact. This implies $g(C)=1$. Applying Lemma 2.4 to the irreducible components of the fibres of $g$ then shows that $g$ has irreducible fibres, as we want.

Lemma 2.6. Let $X$ be a compact Kähler threefold which satisfies (C). Then

$$
c_{1} c_{2}(X)=c_{3}(X)=0 .
$$

Proof. Condition (C) implies immediately $\chi\left(X, \Omega_{X}^{p}\right)=0$ for all $p$ and so the claim follows from Riemann-Roch.

\section{Reduction to minimal threefolds or Mori fibre spaCes}

Proposition 3.1. Let $X$ be a smooth complex projective threefold with a one-form $\omega \in$ $H^{0}\left(X, \Omega_{X}^{1}\right)$ such that $\left(H^{*}(X, \mathbb{C}), \wedge \omega\right)$ is exact. If $K_{X}$ is not nef and $X$ does not carry the structure of a Mori fibre space, then there is a smooth projective threefold $Y$ such that $X$ is the blow-up of $Y$ along a smooth elliptic curve $E$. Moreover, if $\omega^{\prime} \in H^{0}\left(Y, \Omega_{Y}^{1}\right)$ denotes the one-form induced by $\omega$, then $\left(H^{*}(Y, \mathbb{C}), \wedge \omega^{\prime}\right)$ is exact and $\left.\omega^{\prime}\right|_{E}$ is nonzero.

Proof. If $K_{X}$ is not nef and $X$ does not carry the structure of a Mori fibre space, then by [Mor82, Theorem 3.3], there is a divisorial contraction $f: X \rightarrow Y$ whose exceptional divisor $E$ has one of the following two properties:

- $\left.f\right|_{E}: E \rightarrow f(E)$ is a $\mathbb{P}^{1}$-bundle over a smooth curve $C=f(E), Y$ is smooth and $X=B l_{C} Y$

- $f(E)$ is a point.

Moreover, in both cases, $E$ contains a curve which has negative self-intersection with $E$. It thus follows from Lemma 2.4, applied to the Albanese map of $X$, that $E$ cannot be contracted to a point and so $f$ must be the blow-up along a smooth curve $C \subset Y$. The 
formula for the cohomology of blow-ups shows that exactness of $\left(H^{*}(X, \mathbb{C}), \wedge \omega\right)$ implies that $\left(H^{*}(Y, \mathbb{C}), \wedge \omega^{\prime}\right)$ is exact and $\left.\omega^{\prime}\right|_{C}$ is nonzero. This concludes the proposition.

Corollary 3.2. Let $X$ be a smooth complex projective threefold which satisfies condition $(C)$. Then there is a smooth projective threefold $X^{\text {min }}$ with a birational morphism $\sigma$ : $X \rightarrow X^{\mathrm{min}}$, which is given as a sequence of blow-ups along smooth elliptic curves that are not contracted via the natural map to $\operatorname{Alb}\left(X^{\mathrm{min}}\right)$. Moreover, $X^{\mathrm{min}}$ satisfies $(C)$ and it is either minimal or a Mori fibre space.

Proof. This is a direct consequence of Proposition 3.1, where we note that an elliptic curve $E$ on a smooth projective variety $X$ is contracted via the Albanese map of $X$ if and only if any holomorphic one-form on $X$ restricts trivially on $E$.

By Proposition 3.1 and Corollary 3.2, the proof of Theorems 1.2 and 1.3 reduce to the case where $X$ is either minimal, or it admits the structure of a Mori fibre space.

The following corollary of the above discussion generalizes the main result of Luo and Zhang in [LZ05].

Corollary 3.3. A smooth projective threefold $X$ which satisfies condition (A), (B) or (C) is not of general type.

Proof. Since $(\mathrm{A}) \Rightarrow(\mathrm{B})$ is clear and $(\mathrm{B}) \Rightarrow(\mathrm{C})$ by [Sch19] (see Theorem 2.2), we may assume that $X$ satisfies $(\mathrm{C})$. For a contradiction, we assume that $X$ is of general type. By Corollary 3.2, we may additionally assume that $X$ is minimal. By the Miyaoka-Yau inequality,

$$
0>c_{1}^{3}(X) \geq \frac{8}{3} c_{1} c_{2}(X) .
$$

This contradicts Lemma 2.6, which concludes the corollary.

\section{1-FORMS ON THREEFOLDS OF NON-NEGATIVE KODAIRA DIMENSION}

In the case of non-negative Kodaira dimension, our main results will follow from:

Theorem 4.1. Let $X$ be a smooth complex projective threefold of non-negative Kodaira dimension and with $K_{X}$ nef. Assume that $X$ satisfies condition $(C)$.

Then there is a finite Galois étale covering $\tau: X^{\prime} \rightarrow X$ which splits into a product $X^{\prime} \simeq A^{\prime} \times S^{\prime}$, where $A^{\prime}$ is an abelian variety of positive dimension.

The proof of the above theorem occupies the following three sections and the final arguments will be summarized in Section 7. Before we turn to the proofs, let us note the following consequence.

Corollary 4.2. In the notation of Theorem 4.1, let $\omega \in H^{0}\left(X, \Omega_{X}^{1}\right)$ be the one-form from condition $(C)$. Then the following holds: 
(1) $S^{\prime}$ is smooth projective with $\kappa\left(S^{\prime}\right)=\kappa(X)$;

(2) we may up to passing to a finite Galois étale covering of $X^{\prime}$ assume that $A^{\prime}$ is simple and $\tau^{*} \omega$ restricts to a nonzero form on $A^{\prime} \times\{s\}$ for all $s \in S^{\prime}$;

(3) $\tau^{*} \omega$ has no zeros on $X^{\prime}$ and so $\omega$ has no zeros on $X$;

(4) there is a finite group $G \subset \operatorname{Aut}\left(A^{\prime}\right) \times \operatorname{Aut}\left(S^{\prime}\right)$, which acts diagonally and freely on $A^{\prime} \times S^{\prime}$ with quotient $X \simeq\left(A^{\prime} \times S^{\prime}\right) / G$. Moreover, $A:=A^{\prime} / G$ is an abelian variety, $A^{\prime} \rightarrow A$ is étale and the natural map $\pi: X \rightarrow A$ is smooth.

Proof. Since $\tau$ is étale and $X$ is smooth, so is $X^{\prime}$. Hence, $S^{\prime}$ is smooth of Kodaira dimension $\kappa\left(S^{\prime}\right)=\kappa\left(X^{\prime}\right)=\kappa(X)$, as claimed. Up to a finite Galois étale cover, $A^{\prime}$ splits into simple factors and so we may assume that $A^{\prime}$ is simple. Moreover,

$$
\tau^{*} \omega \in H^{0}\left(X^{\prime}, \Omega_{X^{\prime}}^{1}\right) \simeq H^{0}\left(A^{\prime}, \Omega_{A^{\prime}}^{1}\right) \oplus H^{0}\left(S^{\prime}, \Omega_{S^{\prime}}^{1}\right) .
$$

To see that $\tau^{*} \omega$ restricts to a nonzero form on $A^{\prime} \times\{s\}$ for all $s \in S^{\prime}$, it suffices to show that it does not map to zero under the projection to $H^{0}\left(A^{\prime}, \Omega_{A^{\prime}}^{1}\right)$. If it does map to zero, then $S^{\prime}$ satisfies the equivalent conditions $(\mathrm{A}) \Leftrightarrow(\mathrm{B}) \Leftrightarrow(\mathrm{C})$. This implies by [Sch19, Corollary 3.2] that some étale cover of $S^{\prime}$ splits off a positive-dimensional simple abelian variety and the restriction of the pullback of $\omega$ to that factor is non-trivial, as we want. Hence, item (2) of the corollary holds. Moreover, item (3) is an immediate consequence of item (2).

Since $A^{\prime} \times S^{\prime} \rightarrow X$ is a Galois étale cover, there is a finite subgroup $G \subset \operatorname{Aut}\left(A^{\prime} \times S^{\prime}\right)$ with $X \simeq\left(A^{\prime} \times S^{\prime}\right) / G$. By item $(2)$, the sub-Hodge structure $H^{1}\left(A^{\prime}, \mathbb{Q}\right) \subset H^{1}\left(X^{\prime}, \mathbb{Q}\right)$ is simple. Since $\tau^{*} \omega$ restricts nontrivially on $A^{\prime} \times\{s\}$ for all $s \in S^{\prime}$, we conclude that $G$ acts trivially on $H^{1}\left(A^{\prime}, \mathbb{Q}\right) \subset H^{1}(X, \mathbb{Q})$ and hence trivially on $H^{0}\left(A^{\prime}, \Omega_{X}^{1}\right) \subset H^{0}\left(X, \Omega_{X}^{1}\right)$. This implies that for any $s \in S^{\prime}$ and $g \in G$,

$$
g\left(A^{\prime} \times\{s\}\right)=A^{\prime} \times\left\{s^{\prime}\right\}
$$

for some $s^{\prime} \in S^{\prime}$. Hence, $G$ acts on $S^{\prime}$ such that the projection $A^{\prime} \times S^{\prime} \rightarrow S^{\prime}$ becomes $G$-equivariant. For $s \in S^{\prime}$ and $g \in G$, the action of $g$ yields an isomorphism

$$
A^{\prime} \times\{s\} \stackrel{\sim}{\longrightarrow} A^{\prime} \times\{g \cdot s\},
$$

and hence an element $g(s) \in \operatorname{Aut}\left(A^{\prime}\right)$. Since $G$ is a finite group, $g(s)$ must have finite order and so it is locally constant in $s$. Since $S^{\prime}$ is connected, $G$ acts diagonally on $A^{\prime} \times S^{\prime}$. Moreover, $A^{\prime} \rightarrow A:=A^{\prime} / G$ is étale, as it induces an isomorphism on one-forms. It thus follows that $\pi: X \rightarrow A$ is smooth, as we already know that it is smooth after some finite étale cover. This concludes the corollary.

\section{Proof of Theorem 4.1 for $\kappa(X)=2$}

In this section we aim to prove Theorem 4.1 in the case where $\kappa(X)=2$. 


\subsection{Preliminaries on elliptic threefolds.}

Definition 5.1. An elliptic threefold is a normal projective threefold $X$ with a morphism $f: X \rightarrow S$ to a normal projective surface whose general fibre is an elliptic curve. We say that $f$ has trivial (or no) monodromy, if $R^{1} f_{*} \mathbb{Q}$ restricts to a trivial local system over some non-empty (Zariski) open subset $U \subset S$.

Lemma 5.2. Let $f: X \rightarrow S$ be an elliptic threefold with trivial monodromy and such that $X$ has rational singularities (e.g. terminal singularities). Then the general fibre of $f$ is not contracted via the Albanese morphism $a: X \rightarrow \operatorname{Alb}(X)$.

Proof. Since $X$ has rational singularities, the Albanese morphism of any resolution $\tilde{X}$ of $X$ factors through $X$, and so $a$ is defined. The lemma then follows from Deligne's global invariant cycle theorem (see e.g. [Voi03, Theorem 4.24]) applied to $\tilde{X}$, which implies that $\widetilde{X}$ carries a holomorphic one-form which restricts nontrivially on the general fibre of the natural map $\tilde{X} \rightarrow \operatorname{Alb}(\tilde{X})=\operatorname{Alb}(X)$. The latter map factors through $a$, and so the lemma follows.

The following result is due to Grassi [Gra94].

Theorem 5.3. Let $X$ be terminal threefold with $K_{X}$ nef of Kodaira dimension two and with Iitaka fibration $f: X \rightarrow S$. If $c_{1} c_{2}(X)=0$, then $f$ has only finitely many singular fibers which are not multiples of a smooth elliptic curve.

Proof. This follows from the proof of [Gra94, Theorem 2.7]. Indeed, by [Gra94, Corollary 2.3.2], $c_{1} c_{2}(X)=-12\left(K_{S}+\Lambda\right) \Delta$, where $\Delta \subset S$ is the divisor given by the locus of nonmultiple singular fibres of $f$ and $\Lambda$ is a divisor on $S$ with $K_{X}=f^{*}\left(K_{S}+\Lambda\right)$. Since $f$ is the Iitaka fibration, $K_{S}+\Lambda$ is ample and so $c_{1} c_{2}(X)=0$ implies $\Delta=0$. This proves the theorem.

We have the following important structure theorem of Nakayama. To state it, recall that an elliptic threefold $f: X \rightarrow S$ is a standard elliptic fibration if $X$ is $\mathbb{Q}$-factorial and terminal, $f$ is equi-dimensional and $K_{X} \sim_{\mathbb{Q}} f^{*}\left(K_{S}+\Delta\right)$ for an effective $\mathbb{Q}$-divisor $\Delta$ such that $(S, \Delta)$ is log terminal.

Theorem 5.4 ([Nak02, Theorem A.1]). Let $f: X \rightarrow S$ be an elliptic threefold. Then there is a proper birational morphism $S^{\prime} \rightarrow S$ and a standard elliptic fibration $f^{\prime}: X^{\prime} \rightarrow$ $S^{\prime}$ that is birational to $f$ over $S$, such that $K_{X^{\prime}}$ is semi-ample over $S$.

Lemma 5.5. Let $f: X \rightarrow S$ be an elliptic threefold, such that $X$ is terminal, $\mathbb{Q}$-factorial and $K_{X}$ is $f$-nef. Let $S^{\prime} \rightarrow S$ and $f^{\prime}: X^{\prime} \rightarrow S^{\prime}$ be as in Theorem 5.4. Then there is a smooth open subset $U^{\prime} \subset S^{\prime}$, whose complement in $S^{\prime}$ is zero-dimensional, and such 
that the base change $X_{U^{\prime}}^{\prime}:=f^{\prime-1}\left(U^{\prime}\right)$ is a smooth threefold. Moreover, for any such open subset $U^{\prime} \subset S^{\prime}$, the natural birational map $X_{U^{\prime}}^{\prime} \rightarrow X$ induces an isomorphism

$$
\pi_{1}\left(X_{U^{\prime}}^{\prime}\right) \simeq \pi_{1}\left(X^{\mathrm{sm}}\right)
$$

where $X^{\text {sm }} \subset X$ denotes the smooth locus of $X$.

Proof. Since $f^{\prime}$ is a standard elliptic fibration, $X^{\prime}$ has only terminal $\mathbb{Q}$-factorial singularities, $f^{\prime}$ is equi-dimensional and $S^{\prime}$ has only klt singularities. In particular, $X^{\prime}$ and $S^{\prime}$ have isolated singularities. Hence, there is an open subset $U^{\prime} \subset S^{\prime}$ whose complement in $S^{\prime}$ is zero-dimensional, and such that the base change $X_{U^{\prime}}^{\prime}:=f^{\prime-1}\left(U^{\prime}\right)$ is smooth.

Let now $U^{\prime} \subset S^{\prime}$ be any such subset. Since $K_{X^{\prime}}$ is semi-ample over $S$, it follows that $X$ and $X^{\prime}$ are birational minimal models over $S$ and so they are isomorphic in codimension one, see e.g. [KM05, Theorem 3.52(2)]. Since $S^{\prime} \backslash U^{\prime}$ is zero-dimensional and $f^{\prime}$ is equidimensional, $X^{\prime} \backslash X_{U^{\prime}}^{\prime}$ is at most one-dimensional. Since $X^{\prime}$ and $X$ are isomorphic in codimension one, we conclude the same for $X_{U^{\prime}}^{\prime}$ and $X$, and hence also for $X_{U^{\prime}}^{\prime}$ and $X^{\mathrm{sm}}$, because $X$ is terminal and so it has isolated singularities. Since $X_{U^{\prime}}^{\prime}$ and $X^{\text {sm }}$ are smooth, this implies $\pi_{1}\left(X_{U^{\prime}}^{\prime}\right) \simeq \pi_{1}\left(X^{\mathrm{sm}}\right)$, as we want.

\subsection{Condition (C) implies trivial monodromy after étale cover.}

Lemma 5.6. Let $X$ be a smooth projective threefold with $K_{X}$ nef of Kodaira dimension two and with Iitaka fibration $f: X \rightarrow S$. Assume that $(C)$ holds for $X$. If $f$ has non-trivial monodromy, then $f$ is equi-dimensional.

Proof. Assume that $f$ has non-trivial monodromy. Then the general fibre of $f$ is contracted via the Albanese map $a: X \rightarrow \operatorname{Alb}(X)$. This implies that $a$ factors rationally through $f$. Since $S$ is the base of the Iitaka fibration, it has at most klt singularities, hence rational singularities, and so any rational map from $S$ to $\operatorname{Alb}(X)$ is a morphism. That is, $a$ factors through $f$. Hence, any prime divisor $D \subset X$ which maps to a point on $S$ is contracted to a point by the Albanese map of $X$. Lemma 2.4 then shows that $\operatorname{Alb}(X)$ is an elliptic curve and $D$ is numerically equivalent to a rational multiple of a fibre of $a$. Since $D$ is contracted by $f, f$ would need to factor through $a$, which is impossible because $S$ is a surface. This proves the lemma.

Remark 5.7. The condition on the monodromy is necessary in Lemma 5.6. To see this, let $S$ be a canonical surface with ample canonical bundle and a single node as singularity, and with minimal resolution $\tilde{S} \rightarrow S$. Then for any elliptic curve $E$, the product $X:=\tilde{S} \times E$ is a minimal threefold of Kodaira dimension two which satisfies (C), but the Iitaka fibration of $X$ is given by the natural map $X \rightarrow S$, which is not equi-dimensional. 
We are now able to prove the following, which is the main result of Section 5.2.

Proposition 5.8. Let $f: X \rightarrow S$ be an elliptic threefold, with $X$ smooth and $K_{X}$ nef. Assume that $X$ satisfies $(C)$. Then there is a finite étale Galois cover $\tau: \widetilde{X} \rightarrow X$, such that the elliptic fibration $\tilde{f}: \widetilde{X} \rightarrow \widetilde{S}$ that is induced by $f$ has trivial monodromy.

Proof. By Lemma 5.6, we may assume that $f$ is equi-dimensional. By Theorem 5.3, only finitely many singular fibers of $f$ are not multiples of a smooth elliptic curve. Let $B \subset S$ be a general hyperplane section. Then the base change $X_{B} \rightarrow B$ is a minimal elliptic surface all of whose singular fibres are multiple. This implies that any two smooth fibres of $X_{B} \rightarrow B$ are isomorphic. Hence, $f$ is generically isotrivial, with typical fibre a smooth elliptic curve $F$. This implies that there is an open subset $U \subset S$ whose complement is zero-dimensional, such that $\left.R^{1} f_{*} \mathbb{Z}\right|_{U}$ is a local system (which above the multiple fibres can be checked via topological base change). Moreover, this local system has finite monodromy, because the identity component $\operatorname{Aut}^{0}(F)$ of $\operatorname{Aut}(F)$ acts trivially on $H^{1}(F, \mathbb{Q})$.

It follows that there is a finite étale cover $\tilde{U} \rightarrow U$ such that the base change $X \times{ }_{S} \tilde{U}$ is an elliptic threefold over $\tilde{U}$ with trivial monodromy. Note that $X \times_{S} \tilde{U}$ is a finite étale cover of $f^{-1}(U)$. Since $f$ is equi-dimensional $\pi_{1}\left(f^{-1}(U)\right)=\pi_{1}(X)$ and so this finite étale cover extends to a finite étale cover $\tau: \tilde{X} \rightarrow X$, such that the map $\tilde{f}: \tilde{X} \rightarrow \tilde{S}$, induced via Stein factorization of $f \circ \tau: \tilde{X} \rightarrow S$, is an elliptic threefold with trivial monodromy. By Theorem 2.1, we may assume that $\tau$ is Galois, which finishes the proof of the proposition.

Remark 5.9. The case where $X$ is a product of a curve with a bi-elliptic surfaces shows that the étale covering performed in Proposition 5.8 is really necessary.

5.3. Classification of minimal elliptic threefolds with trivial monodromy. Proposition 5.8 reduces the proof of Theorem 4.1 for $\kappa(X)=2$ to the case of elliptic threefolds $X \rightarrow S$ with trivial monodromy. Even though $X$ is smooth in that situation, it is not much harder to classify more generally such threefolds with terminal singularities. This is the content of the following theorem.

Theorem 5.10. Let $f: X \rightarrow S$ be an elliptic threefold with trivial monodromy, where $X$ is terminal and $K_{X}$ is nef. Then there is a finite quasi-étale Galois covering $\tau: X^{\prime \prime} \rightarrow X$ with $X^{\prime \prime} \simeq S^{\prime \prime} \times E$, where $E$ is an elliptic curve and $S^{\prime \prime}$ is a smooth projective surface with a generically finite map to $S$.

Proof. Note that the assumptions on $X$ are preserved under finite quasi-étale cover. By [GKP16, Theorem 1.5], we may thus up to replacing $X$ by some finite quasi-étale Galois covering assume that any finite étale cover of $X^{\text {sm }}$ extends to a finite étale cover of $\mathrm{X}$. 
By Theorem 5.4, there is a birational morphism $S^{\prime} \rightarrow S$ and a standard elliptic fibration $f^{\prime}: X^{\prime} \rightarrow S^{\prime}$ that is birational to $f$ over $S$ and such that $K_{X^{\prime}}$ is nef over $S$. By Lemma 5.5, there is a smooth open subset $U^{\prime} \subset S^{\prime}$ whose complement is zero-dimensional and such that $X_{U^{\prime}}^{\prime}:=f^{\prime-1}\left(U^{\prime}\right)$ is smooth and the birational map $X_{U^{\prime}}^{\prime} \rightarrow X$ induces an isomorphism

$$
\pi_{1}\left(X_{U^{\prime}}^{\prime}\right) \simeq \pi_{1}\left(X^{\mathrm{sm}}\right)
$$

Since $X^{\prime}$ has only terminal singularities, there is a well-defined Albanese map $a^{\prime}: X^{\prime} \rightarrow$ $\operatorname{Alb}\left(X^{\prime}\right)$, obtained by observing that the Albanese map of any desingularization of $X^{\prime}$ factors through $X^{\prime}$. By Lemma 5.2, the general fibre of $f^{\prime}: X^{\prime} \rightarrow S^{\prime}$ is not contracted by $a^{\prime}$. Hence, the general fibre of $f^{\prime}$ is via $a^{\prime}$ mapped to a translate of a fixed elliptic curve $E \subset \operatorname{Alb}\left(X^{\prime}\right)$. Since $X^{\prime}$ is projective, we can dualize this inclusion to get a surjection $\operatorname{Alb}\left(X^{\prime}\right) \longrightarrow E$. Composing this with $a^{\prime}$, we get a surjection

$$
g: X^{\prime} \rightarrow E
$$

which restricts to an isogeny on general fibres of $f^{\prime}$. Taking the Stein factorization, we may assume that $g$ has connected fibres.

Since $X^{\prime}$ is terminal, it has isolated singularities. A general fibre $\tilde{S}=g^{-1}(e)$ of $g$ is therefore smooth and we consider the normalization $\tilde{X}$ of the base change $X^{\prime} \times_{S^{\prime}} \tilde{S}$. We then get a commutative diagram

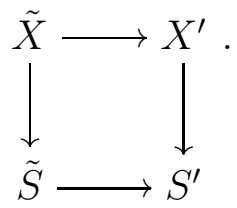

Let $\tilde{U} \subset \tilde{S}$ be the preimage of $U^{\prime} \subset S^{\prime}$. We consider the base change $\tilde{X}_{\tilde{U}} \subset \tilde{X}$. Since $f$ has trivial monodromy by assumptions, the same holds for $f^{\prime}$. Since additionally $K_{X^{\prime}}$ is nef over $S^{\prime}$, the base change $X_{Z}^{\prime}$ to a general hyperplane section $Z \subset S^{\prime}$ is a smooth minimal elliptic surface with trivial monodromy and so all its singular fibres are multiple fibres. Hence, away from finitely many points in $S^{\prime}$, all singular fibres of $f^{\prime}$ are multiple fibres. W.l.o.g., we may assume that this holds for all fibres of $X_{U^{\prime}}^{\prime} \rightarrow U^{\prime}$.

By construction of $\tilde{S} \rightarrow S^{\prime}$, the ramification data of this finite covering corresponds exactly to the locus in $S^{\prime}$ which parametrizes multiple fibres of $f^{\prime}: X^{\prime} \rightarrow S^{\prime}$. It then follows from a local computation, that $\tilde{X}_{\tilde{U}} \rightarrow \tilde{U}$ is a smooth elliptic bundle and $\tilde{X}_{\tilde{U}} \rightarrow$ $X_{U^{\prime}}^{\prime}$ is étale. Up to replacing $\tilde{U}$ by a further finite étale covering, we may by Theorem 2.1 additionally assume that $\tilde{X}_{\tilde{U}} \rightarrow X_{U^{\prime}}^{\prime}$ is a finite Galois étale covering and that the smooth elliptic bundle $\tilde{X}_{\tilde{U}} \rightarrow \tilde{U}$ has trivial monodromy. Since this bundle has a section by construction, the existence of a fine moduli space for elliptic curves with level structure shows that $\tilde{X}_{\tilde{U}} \simeq \tilde{U} \times E$ for an elliptic curve $E$. 
Recall that $\pi_{1}\left(X_{U^{\prime}}^{\prime}\right) \simeq \pi_{1}\left(X^{\mathrm{sm}}\right)$ by $(1)$, and any finite étale cover of $X^{\mathrm{sm}}$ extends to a finite quasi-étale cover of $X$ by the reduction step at the beginning of the proof. Therefore, the finite Galois étale cover $\tilde{X}_{\tilde{U}} \rightarrow X_{U^{\prime}}^{\prime}$ is birational to a finite Galois quasiétale covering

$$
X^{\prime \prime} \rightarrow X
$$

of $X$. Since $\tilde{X}_{\tilde{U}} \simeq \tilde{U} \times E$, we conclude that $X^{\prime \prime}$ is birational to $S^{\prime \prime} \times E$, where $S^{\prime \prime}$ is a minimal surface and $E$ is an elliptic curve. Since $K_{X}$ is nef, so is $K_{X^{\prime \prime}}$. Moreover, $X^{\prime \prime}$ is terminal by [KM05, Proposition 5.20], because it is a finite quasi-étale cover of a terminal threefold.

By $\left[\mathrm{KM} 05\right.$, Theorem 6.25], there is a $\mathbb{Q}$-factorialization $\sigma: \tilde{X}^{\prime \prime} \rightarrow X^{\prime \prime}$, i.e. a proper birational morphism which is an isomorphism in codimension one such that $\tilde{X}^{\prime \prime}$ is $\mathbb{Q}$ factorial, terminal and $K_{\tilde{X}^{\prime \prime}}$ is nef. Hence, $\tilde{X}^{\prime \prime}$ and $S^{\prime \prime} \times E$ are birational minimal models and so they are connected by a sequence of flops (see [Kol89]). Since $S^{\prime \prime} \times E$ does not admit any non-trivial flop, $\tilde{X}^{\prime \prime} \simeq S^{\prime \prime} \times E$. On the other hand, the product $S^{\prime \prime} \times E$ does not admit a small contraction to a terminal threefold (because any rational curve on it maps to a point on the second factor and so it sweeps out a divisor on $S^{\prime \prime} \times E$ ). Hence, $X^{\prime \prime} \simeq \tilde{X}^{\prime \prime}$ and so $X^{\prime \prime} \simeq S^{\prime \prime} \times E$, as we want. This concludes the proof.

5.4. Proof of Theorem 4.1 for $\kappa(X)=2$. Since $X$ is a smooth projective threefold with $K_{X}$ nef, the Iitaka fibration $f: X \rightarrow S$ is a morphism by the abundance conjecture for threefolds, see [Ka92]. This endows $X$ with the structure of an elliptic threefold. By Proposition 5.8, there exists a finite étale Galois covering $X^{\prime} \rightarrow X$, such that $f$ induces an elliptic fibration $f^{\prime}: X^{\prime} \rightarrow S^{\prime}$ without monodromy. Hence, by Theorem 5.10, there is a finite quasi-étale Galois cover $X^{\prime \prime} \rightarrow X^{\prime}$, such that $X^{\prime \prime} \simeq S \times E$ for some smooth projective surface $S$ and an elliptic curve $E$. Since $\kappa\left(X^{\prime \prime}\right)=2$ and $K_{X^{\prime \prime}}$ is nef, $S$ is a minimal surface of general type. Since the composition $X^{\prime \prime} \rightarrow X^{\prime} \rightarrow X$ is a finite quasi-étale Galois covering, there is a finite subgroup $G \subset \operatorname{Aut}(S \times E)$ with

$$
X \simeq(S \times E) / G
$$

Since $X$ is smooth, the $G$-action on $S \times E$ must be free, cf. Section 2.2. This concludes Theorem 4.1 if $\kappa(X)=2$.

Remark 5.11. In a forthcoming paper we classify all good minimal models with $c_{1}^{n-2} c_{2}=$ 0 . Even in the case of smooth minimal threefolds with $\kappa=2$, we find that the condition $c_{1} c_{2}=0$ is strictly weaker than condition $(C)$. In the above proof this is reflected by the fact that we made essential use of condition $(C)$ in the proof of Lemma 5.6 which is used in Proposition 5.8. 


\section{Proof of Theorem 4.1 for $\kappa(X)=1$}

In this Section we aim to prove Theorem 4.1 in the case where $X$ is a minimal smooth projective threefold with $\kappa(X)=1$. By the abundance conjecture (which is known in dimension three, see [Ka92]), the Iitaka fibration of $K_{X}$ yields a morphism $f: X \rightarrow C$ to a smooth projective curve $C$. We may thus consider the diagram

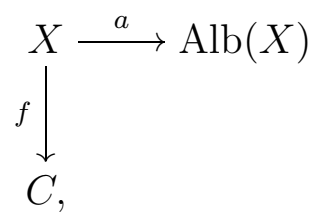

where $a$ denotes the Albanese morphism.

After collecting some preliminary results in Section 6.1, we treat in Sections 6.4, 6.3 and 6.2 below the cases where the Albanese image $a(F) \subset \operatorname{Alb}(X)$ of a general fibre $F$ of $f$ has dimension zero, one and two, respectively.

6.1. Preliminaries. We begin with the following simple observation, cf. [Gra94, Theorem 1.5].

Lemma 6.1. Let $X$ be a smooth projective threefold with Iitaka fibration $f: X \rightarrow C$ to a smooth projective curve $C$. Then, $c_{1} c_{2}(X)=0$ if and only if the smooth fibres of $f$ are bi-elliptic or abelian surfaces.

Proof. Some multiple $m K_{X}$ is linearly equivalent to the pullback of an ample divisor on $C$. Hence, $m K_{X}$ is numerically equivalent to a positive multiple of a general fibre $F$ of $f$. Hence, $c_{1}(X) c_{2}(X)=0$ if and only if $c_{2}(X)$ restricts to zero on $F$, which is to say that $c_{2}(F)=0$. Since $F$ is a minimal surface of Kodaira dimension zero, $c_{2}(F)=0$ implies that $F$ is either bi-elliptic or an abelian surface, see e.g. [BHPV, Chapter VI.1]. This concludes the lemma.

Next, we recall the following definition, see [CaPe00, Definition 1.9].

Definition 6.2. Let $X$ be a smooth projective threefold with a surjective morphism $f$ : $X \rightarrow C$ to a smooth curve $C$. A holomorphic two-form $\eta$ on $X$ is vertical (with respect to $f$ ) if the annihilator of $\eta$ on the tangent space $T_{X, x}$ of $X$ at a general point $x \in X$ is tangential to the fibre of $f$ at $x$.

With this terminology, Campana and Peternell proved the following:

Theorem 6.3 ([CaPe00, Theorem 4.2]). Let $X$ be a minimal smooth projective threefold with $\kappa(X)=1$. Let $f: X \rightarrow C$ be the Iitaka fibration. Assume that there is a holomorphic two-form $\eta$ on $X$ which is not vertical with respect to $f$. Then there is a finite morphism $C^{\prime} \rightarrow C$ such that the normalization $X^{\prime}$ of the base change $X \times_{C} C^{\prime}$ splits into 
a product $X^{\prime} \simeq F \times C^{\prime}$. In particular, $f$ is quasi-smooth, i.e. all singular fibres of $f$ are multiple fibres.

The multiplicities of the singular fibres define an effective divisor $D$ on $C$ and this divisor defines an orbifold structure on $C$, see e.g. [FM94, Section 2.1.3]. We say that such an orbifold is good, if there is an orbifold étale covering with trivial orbifold structure.

Proposition 6.4. Let $X$ be a smooth projective threefold with a morphism $f: X \rightarrow C$ to a smooth projective curve $C$ such that the reduction of each fibre of $f$ is isomorphic to a fixed abelian surface $F$. If the orbifold structure on $C$ that is induced by the multiple fibres of $f$ is good, then there is a Galois étale covering $\tau: X^{\prime} \rightarrow X$ such that $X^{\prime} \simeq F \times C^{\prime}$, where $C^{\prime}$ is a finite cover of $C$.

Proof. If the given orbifold structure is good, then there is a branched cover $C^{\prime} \rightarrow C$, branched exactly at the orbifold points of $C$ with the prescribed multiplicities. The base change $X \times_{C} C^{\prime}$ is singular along the preimage of the singular fibres of $f$. Let $X^{\prime}$ be the normalization of $X \times_{C} C^{\prime}$. Then a local analysis shows that $X^{\prime}$ is smooth, the natural map $\tau: X^{\prime} \rightarrow X$ is étale and $X^{\prime} \rightarrow C^{\prime}$ is smooth. By Theorem 2.1, we may assume that $\tau$ is Galois. Since $X^{\prime}$ is projective, the same argument as in the proof of [CaPe00, Theorem 4.2] shows that up to a further Galois étale base change $X^{\prime} \simeq F \times C^{\prime}$, as we want. ${ }^{1}$ We repeat the argument for convenience of the reader. Consider the natural map $f^{\prime}: X^{\prime} \rightarrow C^{\prime}$ and note that $R^{1} f_{*}^{\prime} \mathbb{Q}$ is a local system. Since $\operatorname{Aut}(F)$ acts on $H^{1}(F, \mathbb{Q})$ via a finite quotient, $R^{1} f_{*}^{\prime} \mathbb{Q}$ has finite monodromy. Hence, after a suitable Galois étale base change, we may assume that $R^{1} f_{*}^{\prime} \mathbb{Q}$ is trivial and so $b_{1}\left(X^{\prime}\right)=b_{1}\left(C^{\prime}\right)+b_{1}(F)$. We thus get a short exact sequence

$$
0 \rightarrow F \rightarrow \operatorname{Alb}\left(X^{\prime}\right) \rightarrow \operatorname{Jac}\left(C^{\prime}\right) \rightarrow 0 .
$$

Since $X^{\prime}$ is projective, this sequence splits after a suitable Galois étale cover of $X^{\prime}$ and so $X^{\prime} \simeq C^{\prime} \times F$, as we want. This concludes the proposition.

We finally recall the classification of all good orbifolds, see e.g. [CHK00, Corollary 2.29].

Theorem 6.5. Let $C$ be a smooth projective curve with an effective divisor $D \in \operatorname{Div}(C)$. The orbifold $(C, D)$ is good unless $C \simeq \mathbb{P}^{1}$ and one of the following holds:

(1) D consists of one point with some multiplicity;

(2) D consists of two points with different multiplicities.

\footnotetext{
${ }^{1}$ Note that in loc. cit. it is claimed that this splitting holds even in the Kähler setting, but this seems to be incorrect, because even after étale cover, the extension (2) does in the non-polarized setting in general not split.
} 
6.2. The general fibre of $f$ is not contracted via the Albanese map. By Lemmas 2.6 and 6.1 , the smooth fibers of $f$ are abelian or bi-elliptic surfaces. Since $\operatorname{dim}(a(F))=2$ in the present case, the smooth fibres must be abelian surfaces. In particular, $a(F) \subset$ $\operatorname{Alb}(X)$ is the translate of a fixed abelian subvariety of $\operatorname{Alb}(X)$. This implies that the pullback of a general holomorphic two-form from $\operatorname{Alb}(X)$ to $X$ is not vertical with respect to $f$. Hence, Theorem 6.3 applies and we see that the reduction of any fibre of $f$ is isomorphic to a fixed abelian surface $F$ and the only singular fibres of $f$ are multiples of $F$. The multiplicities of the singular fibres define an effective divisor $D$ on $C$ and this divisor defines an orbifold structure on $C$, see e.g. [FM94, Section 2.1.3]. We claim that this orbifold structure is good, i.e. there is an orbifold étale covering $C^{\prime} \rightarrow C$ which has trivial orbifold structure. To this end, we may by Theorem 6.5 assume $g(C)=0$. Then the Leray spectral sequence yields $H^{1}\left(X, \mathcal{O}_{X}\right) \simeq H^{0}\left(C, R^{1} f_{*} \mathcal{O}_{X}\right)$. This implies $b_{1}(X)=4$, because a holomorphic one-form on $X$ which vanishes on one smooth fibre of $f$ vanishes at any smooth fibre, since the image of these fibres in $\operatorname{Alb}(X)$ are translates of the same abelian subvariety. Hence, $a: X \rightarrow \operatorname{Alb}(X)$ induces an isogeny on the general fibre of $f$. For a general point $p \in \operatorname{Alb}(X)$, the preimage $C^{\prime}:=a^{-1}(p)$ is a smooth projective curve and the natural map $C^{\prime} \rightarrow C$ is branched exactly at the given points of the orbifold structure on $C$. Hence, the orbifold structure is good, as we want. The proof of Theorem 4.1 in the case treated in Section 6.2 thus follows from Proposition 6.4.

\subsection{The general fibre of $f$ is via the Albanese map contracted to a curve.} Recall that the general fibre of $f$ are either bi-elliptic or abelian surfaces by Lemma 6.1. In the present case, this implies that the fibres of $f$ are mapped via the Albanese morphism $a . X \rightarrow \operatorname{Alb}(X)$ to translates of a fixed elliptic curve $E$ in $\operatorname{Alb}(X)$. Since $X$ is projective, we can dualize the inclusion $E \subset \operatorname{Alb}(A)$ and get a surjection $\operatorname{Alb}(X) \rightarrow E$. This gives rise to a morphism

$$
g: X \rightarrow E,
$$

which when restricted to the fibres of $f$ coincides with $a$ up to isogeny. Taking the Stein factorization, we may assume that $g$ has connected fibres.

By Lemmas 2.6 and 6.1, any smooth fibre $X_{c}$ is either bi-elliptic or an abelian surface. Consider the restriction $\left.g\right|_{X_{c}}: X_{c} \rightarrow E$. A priori, this might not have connected fibres, but taking the Stein factorization, we see that there is a finite Galois étale cover $\tilde{E}_{c} \rightarrow E$, such that $\left.g\right|_{X_{c}}$ factors through a morphism $\tilde{g}_{c}: X_{c} \rightarrow \tilde{E}_{c}$ with connected fibres. Since $X_{c}$ is bi-elliptic or abelian, there is a finite Galois étale cover $\tilde{E}_{c}^{\prime} \rightarrow \tilde{E}_{c}$, such that the induced étale cover $X_{c}^{\prime} \rightarrow X_{c}$ splits into a product

$$
X_{c}^{\prime}=\tilde{E}_{c}^{\prime} \times F_{c},
$$

where $F_{c}$ is an elliptic curve which might depend on $c \in C$. 
Note that $\tilde{E}_{c}^{\prime} \rightarrow E$ is an étale cover and so it is determined by a finite index subgroup of $\pi_{1}(E)=\mathbb{Z}^{\oplus 2}$. In particular, there is a finite étale cover $\tilde{E}^{\prime} \rightarrow E$ which is isomorphic to $\tilde{E}_{c}^{\prime} \rightarrow E$ for all general points $c \in C$. Similarly, there is a finite étale covering $\tilde{E} \rightarrow E$ which is isomorphic to $\tilde{E}_{c} \rightarrow E$ for general $c \in C$.

It is easy to see (e.g. by using an explicit isomorphism $\pi_{1}(E) \simeq \mathbb{Z} \alpha \oplus \mathbb{Z} \beta$ ) that there is a finite Galois étale cover $E^{\prime} \rightarrow E$, such that the following diagram is Cartesian

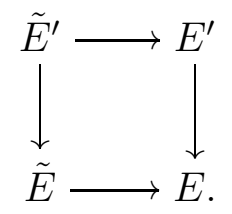

That is, $\tilde{E} \times_{E} E^{\prime} \rightarrow \tilde{E}$ is isomorphic to $\tilde{E}^{\prime} \rightarrow \tilde{E}$.

Let us now consider the finite Galois étale cover $X^{\prime}:=X \times_{E} E^{\prime}$ of $X$ together with the natural maps $g^{\prime}: X^{\prime} \rightarrow E^{\prime}$ and $f^{\prime}: X^{\prime} \rightarrow C$. The fibre of $f^{\prime}$ above a general point $c \in C$ is given by the fibre product

$$
X_{c} \times_{E} E^{\prime} \simeq X_{c} \times_{\tilde{E}_{c}} \tilde{E}_{c}^{\prime}
$$

which coincides with $X_{c}^{\prime}$ from above and so it splits as in (3) into a product $\tilde{E}^{\prime} \times F_{c}$ of elliptic curves. Hence, up to replacing $X$ by $X^{\prime}$, we may assume that for general $c \in C$,

$$
X_{c}=\tilde{E} \times F_{c}
$$

for a finite Galois étale cover $\tilde{E} \rightarrow E$ and for an elliptic curve $F_{c}$ which might depend on $c \in C$.

Note that $\tilde{E} \rightarrow E$ is an isomorphism if and only if $f \times g: X \rightarrow C \times E$ has connected fibres. We show next that we may up to a suitable base change assume that this holds true.

Lemma 6.6. In the above notation, up to replacing $X$ by a finite Galois étale cover, we may assume that $f \times g: X \rightarrow C \times E$ has connected fibres, i.e. $\tilde{E} \rightarrow E$ is an isomorphism.

Proof. To prove the lemma, let $X \stackrel{h}{\longrightarrow} S \longrightarrow C \times E$ be the Stein factorization of $f \times g$. In particular, $S$ is a normal projective surface and we let $\tilde{S} \rightarrow S$ be a minimal resolution. There is a sequence of blow-ups $\tilde{X} \rightarrow X$ along smooth centers, such that the natural rational map $X \rightarrow \tilde{S}$ extends to a morphism $\tilde{X} \rightarrow \tilde{S}$. Since $\tilde{X} \rightarrow \tilde{S}$ has connected fibres, and the natural morphism $\tilde{X} \rightarrow C$ has connected fibres (because $f$ is the Iitaka fibration of $X$ ), we deduce that the natural map $\tilde{S} \rightarrow C$ has connected fibres as well. Let $\tilde{S}_{c}$ and $\tilde{X}_{c}$ be the fibres of $\tilde{S} \rightarrow C$ and $\tilde{X} \rightarrow C$ above $c \in C$. Since $\tilde{X} \rightarrow \tilde{S}$ has connected fibres, $\tilde{X}_{c} \rightarrow \tilde{S}_{c}$ has also connected fibres. Since $\tilde{S}_{c}$ is a curve which maps to $E$, this implies that $\tilde{X}_{c} \rightarrow \tilde{S}_{c}$ is the Stein factorization of the natural map $\tilde{X}_{c} \rightarrow E$. 
Since $\tilde{X}_{c}$ is birational to $X_{c}$ for general $c \in C$, (4) implies

$$
\tilde{S}_{c} \simeq \tilde{E}
$$

That is, $\tilde{S} \rightarrow C$ is an elliptic surface whose general fibres are isomorphic to $\tilde{E} .^{2}$

Note that any fibre $\tilde{S}_{c}$ of $\tilde{S} \rightarrow C$ admits via $\tilde{S} \rightarrow S \rightarrow C \times E$ a natural surjective map to $E$, and so at least one of its components is elliptic. Since $\tilde{S} \rightarrow S$ is the minimal resolution and $S \rightarrow C \times E$ is finite, we conclude that $\tilde{S} \rightarrow C$ is relatively minimal, with typical fibre $\tilde{E}$. Since the arithmetic genus is constant in flat families, we also see that all singular fibres are multiple fibres. This implies that there is a finite Galois étale cover $\tilde{S}^{\prime} \rightarrow \tilde{S}$, such that

$$
\tilde{S}^{\prime} \simeq C^{\prime} \times \tilde{E}
$$

where $C^{\prime}$ is a finite (possibly ramified) cover of $C$ and $\tilde{E}$ is the finite étale cover of $E$ from above.

The cover $\tilde{S}^{\prime} \rightarrow \tilde{S}$ induces via $\tilde{X} \rightarrow \tilde{S}$ a finite Galois étale cover $\widetilde{X}^{\prime} \rightarrow \tilde{X}$, and hence a finite Galois étale cover $X^{\prime} \rightarrow X$, because the proper birational map $\tilde{X} \rightarrow X$ induces an isomorphism $\pi_{1}(\tilde{X}) \simeq \pi_{1}(X)$, as $X$ and $\tilde{X}$ are smooth. By construction, the natural map $X^{\prime} \rightarrow E$ factors through a rational map $X^{\prime} \rightarrow \tilde{E}$, which must be a morphism because $X^{\prime}$ is smooth and $\tilde{E}$ does not contain rational curves. Since $g$ has connected fibres, we conclude that up to replacing $X$ by $X^{\prime}, \tilde{E} \rightarrow E$ must be an isomorphism, as we want. This concludes the proof of the lemma.

Using Lemma 6.6, we assume from now on that $f \times g: X \rightarrow C \times E$ has connected fibres and so it is an elliptic threefold. In particular, $\left.g\right|_{X_{c}}: X_{c} \rightarrow E$ has connected fibres, and so $\tilde{E}=E$ in the above notation, which by (4) yields:

$$
X_{c} \simeq E \times F_{c}
$$

for general $c \in C$, where $F_{c}$ is an elliptic curve that depends on $c$.

By (6), $X_{c}=E \times F_{c}$ for an elliptic curve $F_{c}$ which depends on $c$. Note also that the projection $X_{c} \rightarrow E$ to the first factor coincides with the restriction of $g$ to $X_{c}$, and so it is canonically given. We now aim to compute the generic fibre $X_{\eta}$ of $f: X \rightarrow C$, where $\eta=\operatorname{Spec} k(C)$ denotes the generic point of $C$ (where $k=\mathbb{C}$ ). Over the algebraic closure $\overline{k(C)}, X_{\eta}$ splits by (6) into a product of $E_{\overline{k(C)}}$ with an elliptic curve over $\overline{k(C)}$. The latter must be defined over $k\left(C^{\prime}\right)$ for some finite Galois cover $C^{\prime} \rightarrow C$. That is, there is

\footnotetext{
${ }^{2}$ In particular, the j-invariant of $\tilde{S} \rightarrow C$ is constant, but as the example of Kummer surfaces associated to products of two elliptic curves shows, this alone does not imply that all singular fibres are multiple fibres, contrary to what is claimed in [CaPe00, Lemma 5.2].
} 
a minimal elliptic surface $T \rightarrow C^{\prime}$, and a diagram

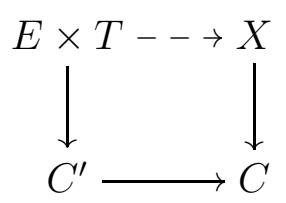

with the property that the horizontal rational map is a morphism over a non-empty open subset $U \subset C$. Moreover, the diagram restricted to the open subset $U \subset C$ is Cartesian. This implies that the Galois group $G$ of the cover $C^{\prime} \rightarrow C$ acts on $E \times T$ birationally in such a way that $X$ becomes birational to $(E \times T) / G$. Explicitly, if $g \in G$ and $c^{\prime} \in C^{\prime}$ lies above a general point $c \in U$, then the action of $g$ identifies $E \times T_{c^{\prime}}$ with $E \times T_{g\left(c^{\prime}\right)}$, by following the isomorphisms in the following diagram

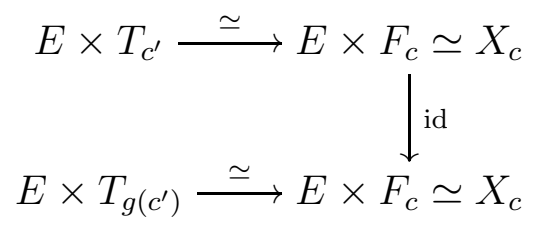

where the horizontal arrows are induced by the upper horizontal arrow in (7) (this yields morphisms because $c \in U)$. Since $\{e\} \times F_{c}$ corresponds to the fibre of $\left.g\right|_{X_{c}}: X_{c} \rightarrow E$ above $e$, it is intrinsically determined and so the isomorphism

$$
E \times T_{c^{\prime}} \stackrel{\simeq}{\longrightarrow} E \times T_{g\left(c^{\prime}\right)}
$$

given by the above diagram respects the product decomposition and acts trivially on the first factor. Hence, $G$ acts on $T$ and we find that $X$ is birational to

$$
(E \times T) / G \simeq E \times(T / G) .
$$

Let $S$ be a minimal model of a resolution of $T / G$. Then $X$ and $E \times S$ are birational minimal models. Hence, they must be connected by flops, see [Kol89]. Since $E \times S$ does not admit any non-trivial flop, $X \simeq S \times E$, as we want. This concludes Theorem 4.1 in Case 2.

6.4. The general fibre of $f$ is via the Albanese map contracted to a point. Since in the present case, the general fibre of $f: X \rightarrow C$ is contracted via $a$, every fibre must be contracted and so the Albanese map factors as $f: X \rightarrow C \rightarrow \operatorname{Jac}(C) \simeq \operatorname{Alb}(X)$. Since $X$ carries a holomorphic one-form $\omega$ such that $\left(H^{*}(X, \mathbb{C}), \wedge \omega\right)$ is exact, $b_{1}(X) \neq 0$ and so $g(C) \geq 1$. Moreover, $\omega$ is a pullback of a one-form from $C$ and so exactness of $\wedge \omega$ shows that $g(C)=1$. Hence, $C \simeq \operatorname{Jac}(C) \simeq \operatorname{Alb}(X)$.

By Lemmas 2.6 and 6.1, the general fibre of $f$ is either an abelian surface or a bi-elliptic surface.

Case 1. $h^{2,0}(X) \neq 0$. 
In this case, $X$ carries a nontrivial holomorphic two-form $\eta$. Since $h^{1,0}(X)=1$, exactness of $\left(H^{*}(X, \mathbb{C}), \wedge \omega\right)$ shows that $\eta \wedge \omega \neq 0$. Since $\omega$ is the pullback of a one-form on $C \simeq \operatorname{Alb}(X)$, the condition $\eta \wedge \omega \neq 0$ then implies that $\eta$ is not vertical. Hence, by Theorem 6.3, the reduction of each fibre of $f$ is isomorphic to the same projective surface $F$, which is either a bi-elliptic or an abelian surface. Since $\eta$ is not vertical with respect to $f$, it restricts to a nonzero form on the general fibre of $f$ and so $F$ must be an abelian surface. The multiple fibres of $f$ give rise to an orbifold structure on $C$ which is good because $g(C) \geq 1$, see Theorem 6.5. Hence, Proposition 6.4 shows that $X$ splits into a product after a finite Galois étale cover, as we want. This proves Theorem 4.1 in the case treated in Section 6.4 if $h^{2,0}(X) \neq 0$.

Case 2. $h^{2,0}(X)=0$ and the general fibre of $f$ is an abelian surface.

In this case, consider the sheaf $f_{*} \omega_{X}$. By Kollár's theorem [Kol86, Theorem I.2.1], this is locally free of rank one, i.e. a line bundle on $C$. Since $h^{3,1}(X)=h^{2,0}(X)$ vanishes, the Leray spectral sequence shows that

$$
H^{1}\left(C, f_{*} \omega_{X}\right)=0
$$

Moreover, $H^{0}\left(C, f_{*} \omega_{X}\right)=0$, as otherwise we get $h^{3,0}(X) \neq 0$, which is impossible because $h^{2,0}(X)=0$ and $\wedge \omega$ is exact on cohomology. It follows that the line bundle $f_{*} \omega_{X}$ has no cohomology and so Riemann-Roch implies that it has degree zero. That is, $f_{*} \omega_{X} \in \operatorname{Pic}^{0}(C)$.

Since $h^{3,0}(X)=0$, the cohomology support locus

$$
\left\{L \in \operatorname{Pic}^{0}(C) \mid H^{0}\left(X, \omega_{X} \otimes f^{*} L\right) \neq 0\right\}
$$

is a union of proper subtori of $\operatorname{Pic}^{0}(C)$, translated by torsion points [Si93]. Since $\operatorname{Pic}^{0}(C)$ is an elliptic curve, the above set is in fact a union of torsion line bundles. This set contains $L:=\left(f_{*} \omega_{X}\right)^{-1} \in \operatorname{Pic}^{0}(C)$, because

$$
H^{0}\left(X, \omega_{X} \otimes f^{*} L\right)=H^{0}\left(C, f_{*} \omega_{X} \otimes L\right)=H^{0}\left(C, \mathcal{O}_{C}\right) \neq 0 .
$$

Hence, $f_{*} \omega_{X} \in \operatorname{Pic}^{0}(C)$ is a torsion line bundle. But then up to passing from $X$ to a suitable Galois étale covering (induced by a Galois étale covering of $C$ ), we may assume that $f_{*} \omega_{X}$ is trivial. This implies $h^{3,0}(X) \neq 0$ and so $h^{2,0}(X) \neq 0$ by exactness of $\left(H^{*}(X, \mathbb{C}), \wedge \omega\right)$. Hence, we may conclude via Case 1 .

Case 3. $h^{2,0}(X)=0$ and the general fibre of $f$ is a bi-elliptic surface.

In this case, $R^{1} f_{*} \omega_{X}=R^{1} f_{*} \omega_{X / C}$ is locally free of rank one. Since $h^{2,0}(X)=h^{3,1}(X)=$ $0, H^{0}\left(C, R^{1} f_{*} \omega_{X}\right)=0$ by the Leray spectral sequence (which degenerates by Kollár's theorem or because $C$ is a curve). Similarly, $b_{1}(X)=b_{1}(C)$ implies $H^{0}\left(C, R^{1} f_{*} \mathcal{O}_{X}\right)=0$. By relative Serre duality,

$$
R^{1} f_{*} \mathcal{O}_{X} \simeq\left(R^{1} f_{*} \omega_{X / C}\right)^{*} \simeq\left(R^{1} f_{*} \omega_{X}\right)^{*},
$$


where we used $\omega_{C}=\mathcal{O}_{C}$ in the last step. Hence, by Serre duality on $C$,

$$
0=H^{0}\left(C, R^{1} f_{*} \mathcal{O}_{X}\right)=H^{1}\left(C, R^{1} f_{*} \omega_{X}\right) .
$$

That is, $R^{1} f_{*} \omega_{X}$ is a line bundle on $C$ without cohomology and so it must be in $\operatorname{Pic}^{0}(C)$.

Since $h^{3,1}(X)=0$, the cohomology support locus

$$
\left\{L \in \operatorname{Pic}^{0}(C) \mid H^{1}\left(X, \omega_{X} \otimes f^{*} L\right) \neq 0\right\}
$$

is a union of torsion line bundles (see [Si93]), where we use that $C$ is an elliptic curve. As before, the Leray spectral sequence $E_{2}^{p, q}=H^{p}\left(C, R^{q} f_{*} \omega_{X}\right) \Rightarrow H^{p+q}(X, \mathbb{C})$ degenerates at $E_{2}$. Hence,

$$
\left\{L \in \operatorname{Pic}^{0}(C) \mid H^{0}\left(C, R^{1} f_{*} \omega_{X} \otimes L\right) \neq 0\right\}
$$

is also a union of torsion line bundles. Since $R^{1} f_{*} \omega_{X}$ is a line bundle of degree zero on $C$, we deduce that it must be torsion. Hence, after a suitable Galois étale base change $C^{\prime} \rightarrow C$, we may assume that $R^{1} f_{*} \omega_{X} \simeq \mathcal{O}_{C}$. This implies $h^{2,0}(X)=h^{1,3}(X) \neq 0$, and so we are done by Case 1 , treated above.

Cases 1, 2 and 3 above finish the proof of Theorem 4.1 in the case treated in Section 6.4. Together with the results in Sections 6.2 and 6.3, this finishes the proof of Theorem 4.1 in the case of Kodaira dimension one.

\section{Proof of Theorem 4.1}

Let $X$ be a smooth projective minimal threefold which satisfies (C) as in Theorem 4.1. By Corollary $3.3 X$ cannot be of general type, and so we are left with the cases $\kappa(X)=0,1,2$.

If $\kappa(X)=2$, then Theorem 4.1 is proven in Section 5.4.

If $\kappa(X)=1$, then the result is proven in Sections 6.2, 6.3 and 6.4 above.

If $\kappa(X)=0, m K_{X}$ is trivial for some $m>0$ by the abundance conjecture for complex projective threefolds, see [Ka92]. It then follows from the Beauville-Bogomolov decomposition theorem (see [Bea83]) and the fact that $b_{1}(X)>0$ that some finite étale cover $\tau: X^{\prime} \rightarrow X$ splits into a product $A \times S$ with a positive-dimensional abelian factor $A$, as required. Here, we may additionally assume that $S$ is simply connected. By Theorem 2.1, up to replacing $X^{\prime}$ by a further étale cover, we may thus assume that $\tau$ is Galois, i.e. a finite étale quotient.

This concludes the proof of Theorem 4.1.

\section{1-FORMS ON MORI FIBER SPACES}

In this Section we prove Theorems 1.2 and 1.3 in the case of negative Kodaira dimension. The main results are Theorems 8.3 and 8.4 below. 
8.1. Preliminaries. Recall the following well-known lemma.

Lemma 8.1. Let $f: X \rightarrow E$ be a surjective morphism from a compact complex manifold $X$ to an elliptic curve $E$. Then the topological Euler characteristic of $X$ is given by

$$
e(X)=\sum_{s \in E}\left(e\left(X_{s}\right)-e\left(X_{g}\right)\right)
$$

where $X_{g}$ denotes a fixed smooth fibre of $f$.

Lemma 8.2. Let $f: X \rightarrow S$ be a surjective morphism from a smooth complex projective threefold $X$ to a smooth projective surface $S$ with $f^{*}: H^{1}(S, \mathbb{C}) \simeq H^{1}(X, \mathbb{C})$. Suppose that for $\omega \in H^{0}\left(S, \Omega_{S}^{1}\right),\left(H^{*}(X, \mathbb{C}), \wedge f^{*} \omega\right)$ is exact. Then $\left(H^{*}(S, \mathbb{C}), \wedge \omega\right)$ is exact.

Proof. Assume first that there is a divisorial contraction $S \rightarrow \bar{S}$ with $\bar{S}$ smooth. Then the composition $g: X \rightarrow \bar{S}$ contracts a divisor $D$ to a point, contradicting Lemma 2.4, applied to the Albanese morphism $a: X \rightarrow \operatorname{Alb}(X)$, because $a$ factors through $S$ by assumptions. Hence, a contraction as above does not exist and so $S$ is minimal. Next, we consider the following diagram

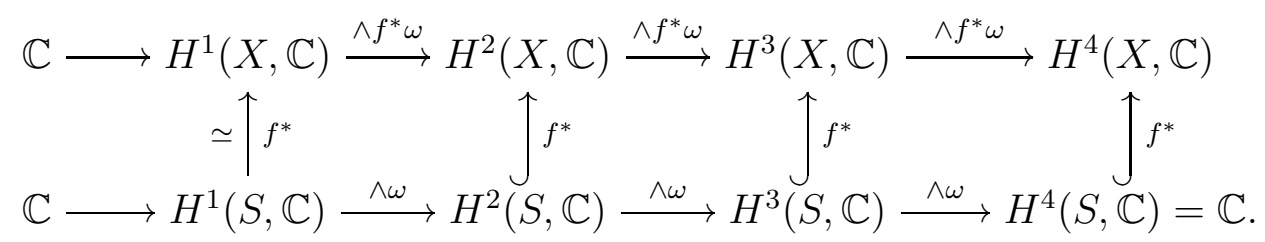

By diagram chasing, we have that $\left(H^{*}(S, \mathbb{C}), \wedge \omega\right)$ is exact at $H^{i}(S, \mathbb{C})$ for $i=0,1,2$. Also, $\left(H^{*}(S, \mathbb{C}), \wedge \omega\right)$ is exact at $H^{4}(S, \mathbb{C})$. Assume $\left(H^{*}(S, \mathbb{C}), \wedge \omega\right)$ is not exact at $H^{3}(S, \mathbb{C})$. Then $c_{2}(S)<0$ and so $S$ is a ruled surfaces over a curve of genus $>1$, see [BHPV, Theorem VI.1.1]. The latter contradicts the fact that the Albanese image of $X$ (and hence that of $S$ ) is fibered by tori by item (2) in Theorem 2.3.

\subsection{Conic bundles over surfaces.}

Theorem 8.3. Let $X$ be a smooth projective threefold which admits the structure of a Mori fibre space $f: X \rightarrow S$ over a projective surface $S$. Let $\omega \in H^{0}\left(X, \Omega_{X}^{1}\right)$ be a holomorphic one-form on $X$ such that for any étale cover $\tau: X^{\prime} \rightarrow X,\left(H^{*}\left(X^{\prime}, \mathbb{C}\right), \wedge \tau^{*} \omega\right)$ is exact. Then the following holds:

(1) $\omega$ has no zero on $X$;

(2) there is a smooth map $\pi: X \rightarrow A$ to a positive-dimensional abelian variety $A$;

(3) $S$ is a smooth projective surface which satisfies $(A) \Leftrightarrow(B) \Leftrightarrow(C)$;

(4) $f$ is either smooth, or $A$ is an elliptic curve and the degeneration locus of $f$ is a disjoint union of smooth elliptic curves which are étale over $A$ (via the map $S \rightarrow A$ induced by $\pi$ ). 
Proof. By [Mor82, Theorem 3.5], $f$ is a conic bundle of relative Picard rank one, $S$ is smooth and the discriminant locus $\Delta_{f}$ of $f: X \rightarrow S$ is a curve with at worst ordinary double points.

Since $f^{*}$ induces an isomorphism on $H^{1}, \operatorname{Alb}(X) \simeq \operatorname{Alb}(S)$ and so we get a commutative diagram

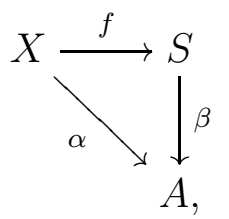

where $A=\operatorname{Alb}(X)=\operatorname{Alb}(S)$ and $\alpha, \beta$ are the respective Albanese morphisms. Hence there is a one-form $\gamma \in H^{0}\left(A, \Omega_{A}^{1}\right)$ with $\omega=\alpha^{*} \gamma=f^{*} \beta^{*} \gamma$.

Let $\tau: S^{\prime} \rightarrow S$ be a finite étale cover, and let $X^{\prime}:=X \times_{S} S^{\prime}$ be the induced étale cover of $X$. Then $X^{\prime} \rightarrow S^{\prime}$ is a conic bundle and so $b_{1}\left(X^{\prime}\right)=b_{1}\left(S^{\prime}\right)$. Hence, Lemma 8.2 implies that $\left(H^{*}\left(S^{\prime}, \mathbb{C}\right), \wedge \tau^{*} \beta^{*} \gamma\right)$ is exact. This proves item (3). In particular, $S$ admits a smooth morphism to an elliptic curve or abelian surface by [Sch19, Corollary 3.2 , which proves item (2) in the case where $f$ is smooth. For what follows, we may thus assume that $\Delta_{f} \neq \emptyset$.

By (3) and [Sch19, Corollary 3.1], $S$ is one of the following:

(a) a minimal ruled surface over an elliptic curve;

(b) an abelian surface;

(c) a minimal elliptic surface $h: S \rightarrow C$ such that one of the following holds:

(i) $h$ is smooth, $C$ is an elliptic curve and $\beta^{*} \gamma \in h^{*} H^{0}\left(C, \Omega_{C}^{1}\right)$;

(ii) $h$ is quasi-smooth, i.e. all singular fibres are multiple fibres, and the restriction of $\beta^{*} \gamma$ to a general fibre of $h$ is non-zero.

By [PaSh99, lemma 7.1.10],

$$
e(X)=2\left(e(S)-p_{a}\left(\Delta_{f}\right)+1\right)
$$

where $p_{a}$ denotes the arithmetic genus of $\Delta_{f}$. Since $e(X)=0$ and $e(S)=0$ by exactness of $\left(H^{*}(X, \mathbb{C}), \wedge \alpha^{*} \gamma\right)$ and $\left(H^{*}(S, \mathbb{C}), \wedge \beta^{*} \gamma\right)$, we deduce

$$
p_{a}\left(\Delta_{f}\right)=1 .
$$

Since $\Delta_{f}$ is a nodal curve, this implies that each connected component of $\Delta_{f}$ is either a smooth elliptic curve with some attached trees of rational curves, or a rational curve with a single node with some attached trees of rational curves. Note that $\Delta_{f}$ cannot contain a smooth $\mathbb{P}^{1}$ which is attached to the remaining components at only one point $p$, as the monodromy of the lines above $\mathbb{P}^{1} \backslash\{p\} \simeq \mathbb{A}^{1}$ would need to be trivial and so $f$ could not have relative Picard rank one, contradicting our assumptions. Moreover, since none of the surfaces in (a)-(cii) contains a rational curve with at least one node, 
we conclude that $\Delta_{f}$ must be a disjoint union of smooth elliptic curves. By assumptions, $\Delta_{f} \neq \emptyset$ and so we pick an irreducible component $D \subset \Delta_{f}$, which is automatically a smooth connected component of $\Delta_{f}$. Hence, each fibre of $f$ above a point $d \in D$ is given by two distinct lines which meet in a point, see e.g. [PaSh99, Proposition 7.1.8(i)].

By [Sch19, Corollary 3.2], some étale cover $S^{\prime} \rightarrow S$ is either a simple abelian surface, or it splits into the product of an elliptic curve with another curve. This implies that there is a finite Galois étale cover $\tau: S^{\prime} \rightarrow S$ whose restriction to $D$ induces an étale cover that trivializes the monodromy of the two lines above points of $D$ in the conic bundle $f: X \rightarrow S$. Let $X^{\prime} \rightarrow X$ be the finite Galois étale cover induced by $S^{\prime}$. Then, $f^{\prime}: X^{\prime} \rightarrow S^{\prime}$ has relative Picard rank at least two, because the monodromy of the two lines above $D^{\prime}:=\tau^{-1}(D)$ is trivial. In particular, $X^{\prime}$ admits a divisorial contraction where exactly one of the two families of lines above $D^{\prime}$ is contracted, and we exhibit $X^{\prime}$ as the blow-up $X^{\prime} \simeq B l_{D^{\prime}} Y$ of a conic bundle $Y \rightarrow S^{\prime}$ which is smooth above $D^{\prime} \subset S^{\prime}$. By Proposition 3.1, the pullback $\tau^{*} \beta^{*} \gamma$ restricts nontrivially on $D^{\prime}$. This implies that $\beta^{*} \gamma$ restricts nontrivially on $D$. Hence, there is a surjective morphism $p: A \longrightarrow E$ to an elliptic curve $E$, so that the composition $p \circ \beta: S \rightarrow E$ restricts on $D$ to an isogeny $D \rightarrow E$.

Since $S$ splits into a product after some étale cover by [Sch19, Corollar 3.2(e)], one checks that $p \circ \beta: S \rightarrow E$ must be a smooth morphism. Since the remaining components of $\Delta_{f}$ are all smooth elliptic curves that are disjoint from $D$, we conclude that in fact any component of $\Delta_{f}$ is étale over $E$ via the morphism $p \circ \beta$. This concludes item (4). Moreover, it shows that the composition $p \circ \beta \circ f: X \rightarrow E$ is smooth, which proves item (2).

It remains to prove item (1). We have seen that the one-form $\omega=f^{*} \beta^{*} \gamma$ comes from a form $\beta^{*} \gamma$ that has no zeros on $S$ by [Sch19, Corollary 3.1]. Hence, $\omega$ has no zeros on $X$ in the case where $f$ is smooth. If $f$ is not smooth, we have seen above that $\beta^{*} \gamma$ restricts nontrivially on each component of the ramification locus $\Delta_{f}$, and this implies that $\omega$. This proves item (1), which finishes the proof of Theorem 8.3.

\subsection{Del Pezzo fibrations.}

Theorem 8.4. Let $X$ be a smooth complex projective threefold which admits a holomorphic one-form $\omega$ such that for any étale cover $\tau: X^{\prime} \rightarrow X,\left(H^{*}\left(X^{\prime}, \mathbb{C}\right), \wedge \tau^{*} \omega\right)$ is exact. Suppose that $X$ admits the structure of a Mori fibre space $f: X \rightarrow C$ over a curve $C$. Then $C$ is an elliptic curve and $f$ is smooth. In particular, $\omega$ has no zeros on $X$.

Proof. By [Mor82, Theorem 3.5.2], $C$ is a smooth projective curve and any fibre of $f$ is an irreducible reduced del Pezzo surface which is Gorenstein, as its canonical bundle is given by the restriction of the canonical bundle of $X$. Moreover, since $\omega$ has its zeros 
exactly at the singular points of the fibres of $f$, and since it cannot have isolated zeros by item (1) in Theorem 2.3, the singular fibres of $f$ are reduced and non-normal.

Note that $f^{*}$ induces an isomorphism $H^{1}(C) \simeq H^{1}(X)$. Since $\left(H^{*}(X, \mathbb{C}), \wedge \omega\right)$ is exact, we deduce that $C$ is an elliptic curve. It remains to prove that $f$ is smooth.

Since $\rho(X / C)=1$ and $h^{2,0}(X)=0, b_{2}(X)=b_{2}(C)+1=2$. Since $b_{1}(X)=b_{1}(C)=2$ and $\left(H^{*}(X, \mathbb{C}), \wedge \omega\right)$ is exact, we then conclude

$$
b_{1}(X)=b_{5}(X)=2, \quad b_{2}(X)=b_{4}(X)=2, \quad b_{3}(X)=2 .
$$

We consider the Leray spectral sequence

$$
E_{2}^{p, q}=H^{p}\left(C, R^{q} f_{*} \mathbb{C}\right) \Rightarrow H^{p+q}(X, \mathbb{C}) .
$$

Since $f$ has connected fibres, $f_{*} \mathbb{C}=\mathbb{C}$. Since the general fibre of $f$ is a smooth del Pezzo surface, $R^{1} f_{*} \mathbb{C}$ is a skyscraper sheaf. Since $E_{\infty}^{1,0}=H^{1}(C, \mathbb{C})$, we deduce $0=E_{\infty}^{0,1}=$ $E_{2}^{0,1}=H^{0}\left(C, R^{1} f_{*} \mathbb{C}\right)$ and so $R^{1} f_{*} \mathbb{C}=0$ is trivial. Hence,

$$
E_{2}^{2,0}=H^{2}\left(C, f_{*} \mathbb{C}\right) \simeq \mathbb{C}, \quad E_{2}^{1,1}=0 \text { and } E_{2}^{0,2}=H^{0}\left(C, R^{2} f_{*} \mathbb{C}\right) .
$$

Analysing the differentials $d_{r}: E_{r}^{p, q} \rightarrow E_{r}^{p+r, q-r+1}$, we find that $E_{2}^{2,0}=E_{\infty}^{2,0}$ and $E_{2}^{0,2}=$ $E_{\infty}^{0,2}$. Since $b_{2}(X)=2$, this implies

$$
H^{0}\left(C, R^{2} f_{*} \mathbb{C}\right)=E_{2}^{0,2}=E_{\infty}^{0,2} \simeq \mathbb{C} .
$$

In particular, $H^{0}\left(C, R^{2} f_{*} \mathbb{C}\right)$ is generated by the section of $R^{2} f_{*} \mathbb{C}$ induced by an ample class on $X$.

By topological proper base change, we have for any $c \in C$ and any $i \in \mathbb{N}$ :

$$
\left(R^{i} f_{*} \mathbb{C}\right)_{c} \simeq H^{i}\left(X_{c}, \mathbb{C}\right) .
$$

We claim that this implies that $b_{2}\left(X_{c}\right)$ is bounded from above by the second Betti number of a smooth fibre of $f$. Indeed, if not, then there is a neighbourhood $U$ of $c \in C$ and a section $s \in R^{2} f_{*} \mathbb{C}(U)$ which is nonzero at the stalk at $c$ but zero at all other stalks. But then $s$ can be extended to a nontrivial global section of $R^{2} f_{*} \mathbb{C}$ which is zero away from the point $c$, contradicting (8).

Let $t \in C$ be a general point, so that the fibre $X_{t}$ is smooth. For any $c \in C$, we have seen above that $b_{2}\left(X_{c}\right) \leq b_{2}\left(X_{t}\right)$. Moreover, since $X_{c}$ is irreducible, $b_{0}\left(X_{c}\right)=b_{4}\left(X_{c}\right)=1$ and so the topological Euler characteristics satisfy

$$
e\left(X_{c}\right) \leq e\left(X_{t}\right)
$$

for all $c \in C$. Since $C$ and $X$ have trivial topological Euler characteristic (cf. Lemma 2.6), we deduce from Lemma 8.1 that

$$
e\left(X_{c}\right)=e\left(X_{t}\right)
$$


for all $c \in C$. In particular,

$$
b_{2}\left(X_{c}\right)=b_{2}\left(X_{t}\right)
$$

for all $c \in C$ and so the stalks of $R^{2} f_{*} \mathbb{C}$ have the same dimension at all points of $C$. This implies that $R^{2} f_{*} \mathbb{C}$ is a local system, because we have seen above that any local section of $R^{2} f_{*} \mathbb{C}$ vanishes if it vanishes generically.

We claim that in order to prove that $f: X \rightarrow C$ is smooth, we may replace $X$ by any finite étale cover, induced by a finite étale cover $C^{\prime} \rightarrow C$. To prove this, let $\tau: X^{\prime} \rightarrow X$ by a finite étale cover, induced by a finite étale cover $C^{\prime} \rightarrow C$. Then $f^{\prime}: X^{\prime} \rightarrow C^{\prime}$ induces an isomorphism on $H^{1}$ and so any holomorphic one-form on $X^{\prime}$ comes from $C^{\prime}$. Similarly, any holomorphic one-form on $X$ comes from $C$. Since $C$ and $C^{\prime}$ are elliptic curves, we deduce that $f$ (resp. $f^{\prime}$ ) is smooth if and only if $X$ (resp. $X^{\prime}$ ) carries a one-form without zeros. Since $\tau: X^{\prime} \rightarrow X$ induces an isomorphism on $H^{1}$, and because a one-form on $X$ has zeros if and only if its pullback via the finite étale map $\tau$ has zeros, we conclude that $f$ is smooth if and only if $f^{\prime}$ is smooth, as claimed.

Since $R^{2} f_{*} \mathbb{C}$ is generically a polarized variation of Hodge structure of type $(1,1)$, its monodromy representation is finite. This implies that up to replacing $X$ by a finite étale covering, we may assume that $R^{2} f_{*} \mathbb{C}$ is trivial. Applying Proposition 3.1 to this base change repeatedly, we may assume that we again arrive at the situation of a Mori fibre space over $C$ and so $\rho(X / C)=1$. Since $R^{2} f_{*} \mathbb{C}$ is trivial, $\rho(X / C)=1$ implies that it has rank one and so the general fibre of $f$ must be $\mathbb{P}^{2}$ (because it is a smooth del Pezzo surface with $b_{2}=1$ ). It is not hard to prove directly that this implies that $f$ cannot have any non-normal fibres; alternatively, this claim also follows from [Fuj90, Theorem 3.1]. Since we have seen above that all singular fibres of $f$ are irreducible reduced non-normal Gorenstein surfaces, this proves that $f$ is smooth. This finishes the proof of Theorem 8.4 .

\section{Summary of the ARgument}

Proof of Theorems 1.1 and 1.2. Since $1.2 \Rightarrow 1.1$, it suffices to prove Theorem 1.2. Since $(\mathrm{D}) \Rightarrow(\mathrm{A}) \Rightarrow(\mathrm{B})$ is clear and $(\mathrm{B}) \Rightarrow(\mathrm{C})$ is proven in [Sch19, Theorem 1.2] in arbtirary dimension, it suffices to prove $(C) \Rightarrow(D)$ for smooth projective threefolds. For this, let $X$ be a smooth projective threefold and assume that $(\mathrm{C})$ holds for the holomorphic one-form $\omega$ on $X$.

By Corollary 3.2, the minimal model program for $X$ yields a smooth projective threefold $X^{\text {min }}$ and a proper birational morphism $\sigma: X \rightarrow X^{\text {min }}$ which is a sequence of blowups along elliptic curves which are not contracted by the natural map to $\operatorname{Alb}\left(X^{\mathrm{min}}\right)$. This proves item (D1). 
To prove items (D2), (D3) and (D4), we may replace $X$ by $X^{\text {min }}$ and assume that $X$ is either minimal or a Mori fibre space. The assertions then follow from Theorem 4.1, Corollary 4.2, and Theorems 8.3 and 8.4.

This concludes the proof of Theorems 1.1 and 1.2 .

Proof of Theorem 1.3. By Proposition 3.1, it suffices to prove Theorem 1.3 for a smooth projective threefold $X$ which is either minimal or a Mori fibre space. In the latter case, Theorem 1.3 follows from Theorems 8.3 and 8.4; in the former case, Theorem 1.3 follows from item (3) in Corollary 4.2. This concludes the proof of Theorem 1.3.

Proof of Corollary 1.5. Let $f: X \rightarrow A$ be a smooth morphism from a smooth projective threefold $X$ with $\kappa(X) \geq 0$ to an abelian variety $A$. If $A$ is zero-dimensional, the claim is trivial and so we may assume that $\operatorname{dim} A>0$. Hence, $X$ carries a holomorphic oneform without zeros and so the equivalent conditions $(\mathrm{A}) \Leftrightarrow(\mathrm{B}) \Leftrightarrow(\mathrm{C}) \Leftrightarrow(\mathrm{D})$ hold by Theorem 1.2.

If $X$ is not minimal, then by Proposition 3.1, $X$ is the blow-up $B l_{C} Y$ of a smooth projective threefold $Y$ along an elliptic curve $C \subset Y$. Since $f: X \rightarrow A$ factors through $Y$, this implies that $A$ must be an elliptic curve and $C$ must be étale over $A$ ( $f$ is nonflat if $\operatorname{dim} A=3$, and it has singular fibres if $\operatorname{dim} A=2$ or if $\operatorname{dim} A=1$ and $C$ is not étale over $A$ ). Hence, up to replacing $X$ by $Y$, we may inductively assume that $X$ is minimal. We claim that under this assumption, $f$ is an analytic fibre bundle. To prove this, we may replace $X$ by any finite étale cover. Hence, by (D3), we may assume that $X=A^{\prime} \times S^{\prime}$ is a product of an abelian variety $A^{\prime}$ of positive dimension and a smooth projective variety $S^{\prime}$. Then for any $s \in S^{\prime}, A^{\prime} \times\{s\}$ maps to a translate of a fixed abelian subvariety $A^{\prime \prime} \subset A$. Let $B:=A / A^{\prime \prime}$. If $\operatorname{dim} B=0$, then we are done. Otherwise, for any $a \in A^{\prime}$, consider the natural composition

$$
S^{\prime}=S^{\prime} \times\{a\} \hookrightarrow S^{\prime} \times A^{\prime}=X \stackrel{f}{\longrightarrow} A \longrightarrow A / A^{\prime \prime}=B,
$$

which is smooth, because $f$ is smooth. Since $\operatorname{dim} B>0$, [Sch19, Corollary 3.2] implies that up to a further étale covering, $S^{\prime}$ splits of a positive-dimensional abelian variety as a direct factor. Argueing as before therefore concludes the proof of the corollary by induction on the dimension.

\section{ACKnowledgement}

The second author thanks Dieter Kotschick for sending him the preprint [Kot13] in spring 2013, where he poses the problem about the equivalence of (A) and (B). We are grateful for useful comments and conversations to Antonella Grassi, Thomas Peternell, Mihnea Popa and Christian Schnell. Both authors are supported by the DFG grant "Topologische Eigenschaften von Algebraischen Varietäten" (project no. 416054549). 


\section{REFERENCES}

[Bea83] A. Beauville, Variétés Kähleriennes dont la première classe de Chern est nulle, J. Differential Geom. 18 (1983), no. 4, 755-782.

[BHPV] W. Barth, K. Hulek, C.A.M. Peters and A. van de Ven, Compact complex surfaces, Springer, Berlin 2004.

[Ca74] J.B. Carrell, Holomorphic one forms and characteristic numbers, Topology 13 (1974), 225-228.

[CaPe00] F. Campana, T. Peternell, Holomorphic 2-forms on complex threefolds, J. Alg. Geom. 9 (2000), 223-264.

[CHK00] D. Cooper, C.D. Hodgson and S.P. Kerckhoff, Three-dimensional orbifolds and cone-manifolds, MSJ Memoirs 5, Tokyo: Mathematical Society of Japan (MSJ), 2000.

[FG65] W. Fischer and H. Grauert, Lokal-triviale Familien kompakter komplexer Mannigfaltigkeiten, Nachrichten der Akademie der Wissenschaften in Göttingen 6 (1965), 90-94

[FM94] R. Friedman and J.W. Morgan, Smooth four-manifolds and complex surfaces, Ergebnisse der Mathematik und ihrer Grenzgebiete (3), 27., Springer-Verlag, Berlin, 1994.

[Fuj90] T. Fujita, On del Pezzo fibrations over curves, Osaka J. Math. 27 (1990), 229-245.

[GL87] M. Green and R. Lazarsfeld, Deformation theory, generic vanishing theorems, and some conjectures of Enriques, Catanese and Beauville, Invent. Math. 90 (1987), 389-407.

[Gra94] A. Grassi, On a question of J. Kollár, Contemporary Mathematics, 162 (1994), 209-214.

[GKP16] D. Greb, S. Kebekus and T. Peternell, Étale fundamental groups of klt spaces, flat sheaves, and quotients of Abelian varieties, Duke Math. J. 165 (2016), 1965-2004.

[HK05] C.D. Hacon and S.J. Kovacs, Holomorphic one-forms on varieties of general type, Ann. Sci. École Norm. Sup. 38 (2005), 599-607.

[Ka92] Y. Kawamata, Abundance theorem for minimal threefolds, Invent. Math. 108 (1991), 229-246.

[KMM94] S. Keel, K. Matsuki and J. M'Kernan, Log abundance theorem for threefolds, Duke Math. J. 75 (1994), 99-119.

[Kot13] D. Kotschick, Holomorphic one-forms, fibrations over the circle, and characteristic numbers of Kähler manifolds, Preprint 2013.

[Kol86] J. Kollár, Higher direct images of dualizing sheaves I, II, Annals of Math., 123(1986), 11-42. 124 (1986), 171-202.

[Kol89] J. Kollár, Flops, Nagoya Math. J. 113 (1989), 15-36.

[Kol93] J. Kollár, Shafarevich maps and plurigenera of algebraic varieties, Invent. Math. 113 (1993), $177-215$.

[Kol94] J. Kollár, Log surfaces of general type; some conjectures, Contemporary Mathematics, 162 (1994), 261-275.

[Kol97] J. Kollár, Singularities of pairs, in Algebraic Geometry, Santa Cruz 1995, Proc. Symp. Pure Math. Amer. Math. Soc. 621997.

[KM05] J. Kollár and S. Mori, Birational geometry of algebraic varieties, Cambridge University Press, Cambridge, 2008.

[LZ05] T. Luo and Q. Zhang, Holomorphic forms on threefolds, Recent progress in arithmetic and algebraic geometry, Contemp. Math. 386, Amer. Math. Soc., Providence, RI, (2005), 87-94.

[Mi87] Y. Miyaoka, The Chern classes and Kodaira dimension of a minimal variety, Alg. Geom. Sendai 1985, Adv. Stud. Pure Math., 10 (1987), 449-476. 
[Mor82] S. Mori, Threefolds whose canonical bundles are not numerically effective, Ann. of Math. 116 (1982), 133-176.

[Nak02] N. Nakayama, Local Structure of an Elliptic Fibration, Higher Dimensional Birational Geometry, 185-295, in: Adv. Studies in Pure Math. 35, 2002.

[PaSh99] A.N. Parshin, I.R. Shafarevich, Algebraic Geometry V: Fano Varieties, Encyclopaedia of Mathematical Sciences, (1999).

[PS14] M. Popa and C. Schnell, Kodaira dimension and zeros of holomorphic one-forms, Ann. of Math. 179 (2014), 1109-1120.

[Sch19] S. Schreieder, Zeros of holomorphic one-forms and the topology of Kähler manifolds, with an Appendix written jointly with Hsueh-Yung Lin, Preprint 2019.

[Si93] C. Simpson, Subspaces of moduli spaces of rank one local systems, Ann. Sci. École Norm. Sup. 26 (1993), 361-401.

[Ti70] D. Tischler, On fibering certain foliated manifolds over $S^{1}$, Topology 9 (1970), 153-154.

[Ue75] K. Ueno, Classification theory of algebraic varieties and compact complex spaces. Lecture Notes in Math. 439, Springer 1975.

[Voi03] C. Voisin, Hodge Theory and Complex Algebraic Geometry II, Cambridge University Press, Cambridge, 2003.

[Za97] Q. Zhang, Global holomorphic one-forms on projective manifolds with ample canonical bundles, J. Alg. Geom. 6 (1997), 777-787.

Mathematisches Institut, LMU München, Theresienstr. 39, 80333 MÜnchen, Germany

E-mail address: Feng.Hao@mathematik.uni-muenchen.de

Mathematisches Institut, LMU München, Theresienstr. 39, 80333 MÜnchen, Germany

E-mail address: schreieder@math.lmu.de 